LBL- -32430

DE92 041201

\title{
Separation of Compounds with Multiple -OH Groups from Dilute Aqueous Solutions via Complexation with Organoboronate
}

Tina Kuo Fung Chow

M.S. Thesis

Department of Chemical Engineering

University of California

and

Energy and Environment Division

Lawrence Berkeley Laboratory

University of California

Berkeley, CA 94720

May 1992

This work was supported by the Director, Office of Energy Research, Office of Basic Energy Sciences, Division of Energy Conversion and Utilization Technology (ECUT), of the U.S. Department of Energy under Contract No. DE-AC03-76SF00098. 


\title{
SEPARATION OF COMPOUNDS WITH MULTIPLE -OH GROUPS FROM DILUTE AQUEOUS SOIUTIONS VIA COMPLEXATION WITH ORGANOBORONATES
}

\author{
Tina kuo Fung Chow
}

\section{ABSTRACT}

This work examines the separation of polar solutes with multiple -OH groups from dilute aqueous solution into an organic phase. The extraction is enhanced by incorporating into the organic phase an agent that complexes reversibly with the solute of interest. The extractant agent investigated in this work is 3-nitrophenylboronic acid (NPBA) in its anionic form $\left(N P B^{-}\right)$. The NPBA, together with Aliquat 336, is dissolved into 2-ethyl-1-hexanol, and the extractant is contacted with aqueous $\mathrm{NaOH}$ to convert NPBA to $\mathrm{NPB}^{-}$. Aliquat 336 is a quaternary amine which provides the counter-ion (TOMA') for the anionic $\mathrm{NPB}^{-}$-solute complex in the organic phase.

The solutes investigated were: 1,2-propanediol, glycerol, fructose, sorbitol and lactic acid. Batch extraction experiments were performed at $25^{\circ} \mathrm{C}$. Partition coefficients, distribution ratios and loadings are reported for varying concentrations of the solute and the NPB-AIl of the solutes investigated showed complexation with $\mathrm{NPB}^{-}$and a.l of the complexes appear to consist of only one NPB' per complex. Ry fitting the extraction data to a theoretical model, the corresponding complexation equilibrium constants were estimated for some of the solutes investigated.

The 1:1 complexation constants for the solutes glycerol, 
fructose and sorbitol follow trends similar to those reported earlier for complexation with $\mathrm{B}(\mathrm{OH})_{4}$; in the aqueous phase; i.e. the complexation constants increase with increasing number of -OH groups available for complexation. This supports the postulate that $-\mathrm{OH}$ groups are involved in the complexation. The assumption of 1:1 complex appears not to be valid for 1,2-propanediol, which showed overloading (more than one mole of solute complexed to one mole NPB') at higher equilibrium aqueous solute concentrations. The -OH group on the $\mathrm{NPB}^{-}$which is left uncomplexed after one solute molecule had bound to the other two $-\mathrm{OH}$ groups may be responsible for the overloading. Overloading is also observed in extraction of lactic acid, but through a different mechanism. It was found that TOMA $^{+}$can extract lactic acid to an extent comparable to the uptake of lactic acid by $\mathrm{NPB}^{-}$. The complexation is probably through formation of an acid-base ion pair.

Losses of NPBA into the aqueous phase during the initial treatment and during extraction could lead to problems and/or poor economics in an inrustrial separation process. One way of overcoming this problam would be to incorporate the NPBA onto a solid support. 
To Mum \& Adrian 


\section{ACKNOWLEDGEMENTS}

First of all, I would like to give my most sincere thanks to my research advisor, Professor c. Judson King, for his guidance and encouragement throughout this project. He has been an excellent mentor to me, without his advice and insights, I would not be able to finish this project so smoothly.

I would also like to thank Professor Chakraborty and Professor Novak who served on my thesis committee and provided helpful advises on preparation of the manuscript.

I am grateful to the members of my research group for friendships and technical advices. Thanks to Lucy for getting me started; Jack and Iisa for warm assistance and answering numerous stupid questions along the way. Thanks to Scott, Jorge and Jane for keeping me company, life in the lab would have been dull without them. Thanks to Judie, Professor King's administrative assistant for keeping us organized.

Lastly, I would like to give special thanks to Mum and Adrian, whose constant love and support from far away sustained me throughout my time here in Berkeley. 


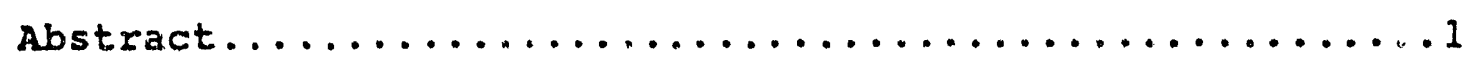

Dedication...............................

Acknowledgments $\ldots \ldots \ldots \ldots \ldots \ldots \ldots \ldots \ldots \ldots \ldots \ldots \ldots \ldots \ldots \ldots$

Table of contents...........................

List of Tables.............................

List of Figures..........................

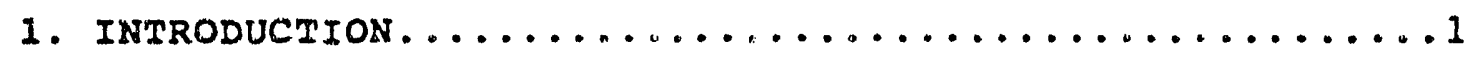

1.1 Chemicals from Biomass.....................

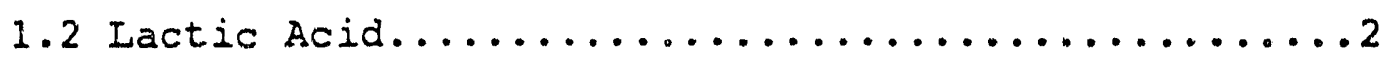

1.3 Propylene Glycol.......................4

1.4 Glycerol.............................

1.5 sorbitol.............................

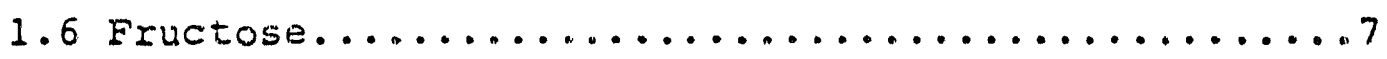

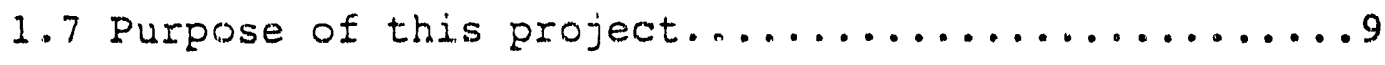

2. SELECTION OF EXTRACTANT SYSTEM................ 11

3. EXPERTMENTAL ........................... 15

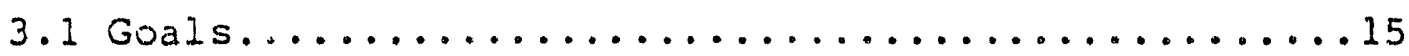

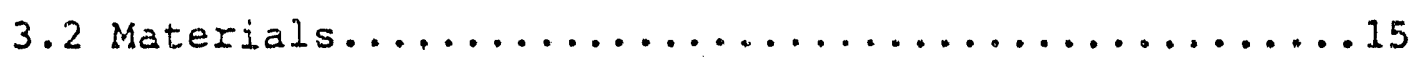

3.3 purity of Materials....................... 17

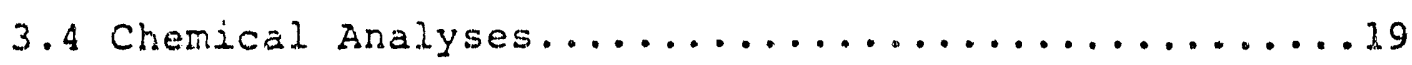


3.4.1 Elemental analyses....................19

3.4 .2 water........................... 19

3.4 .3 Solutes in aqueous solutions............19

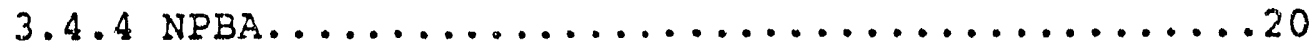

3.5 Procedure............................. 20

3.5.1 Preparation of the Organic Extractant......20

3.5 .2 Forward Extraction..................2

3.5.3 Back Extraction....................24

3.5.4 Effect of Diluent..................24

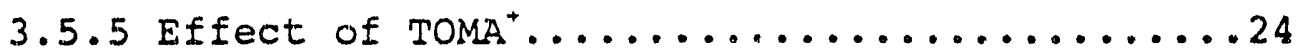

4. TREATMENT OF THE ORGANIC EXTRACTAN'.............26

4.1 Sample calculation........................ 8

4.2 Converting TOMA ${ }^{+} \mathrm{Cl}^{*}$ to $\mathrm{TOMA}^{+} \mathrm{OH}^{-}$first..........29

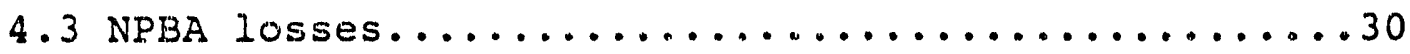

5. NUMERTCAL TREATMENT OF DATA...................

5.1 Experimentally measured quantities............31

5.2 Amount of solute extracted into the organic phase...................................

5.3 Correction for water co-extraction.............32

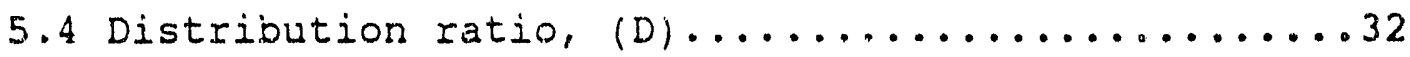

5.5 Partition coefficient, (P).................. 34

5.6 Correction for extraction by diluent...........34

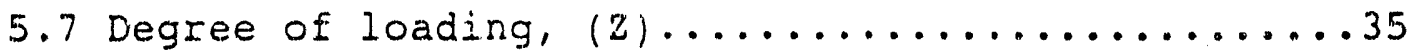


6. CHEMICAL MODELLING OF EXTRACTION DATA.............. 37

7. RESUITS AND DISCUSSION.................... 40

7.1 Partition coefficients and Distribution ratios....40

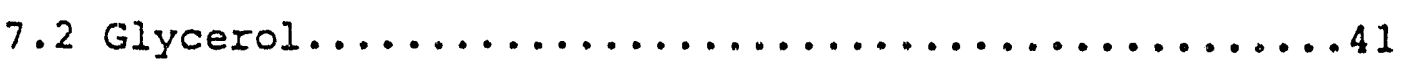

7.3 Iiterature data........................47

7.4 Fructose and sorbitol...................48

$7.51,2$-Propanediol.......................53

7.5 .1 Forward extraction..................53

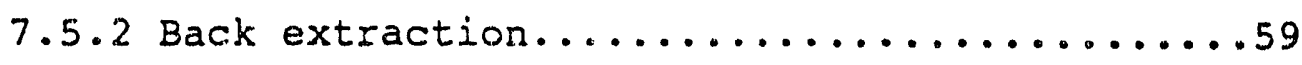

7.5 .3 Effect of diluent................61

7.6 Lactic acid........................6 63

7.7 Discussion.........................67

7.8 Recovery.............................69

7.9 Implementation..........................

REFERENCES. . . . . . . . . . . . . . . . . . . . 71

APPENDIX............................... 


\section{IIST OF TABLES}

Table

page

1. I Characteristics of molasses-based distillery

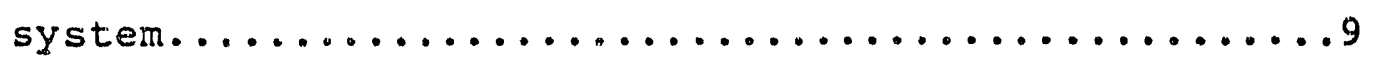

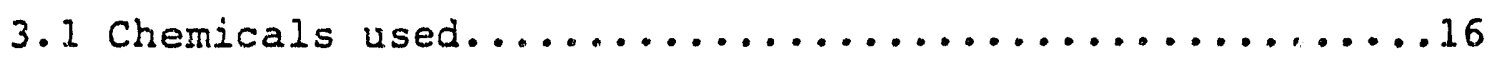

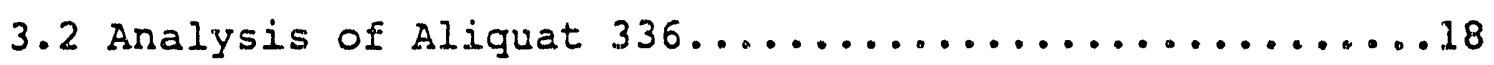

3.3 Components if Organic Extractant................21

4.1 Results of boron and $\mathrm{Cl}^{-}$analysis of the wash solutions for one typical batch of extractant......26

$4.2 \mathrm{pH}$ and $\mathrm{Cl}^{-}$analysis of wash solutions for converting $\mathrm{TOMA}^{+} \mathrm{Cl}^{-}$to $\mathrm{TOMA}^{+} \mathrm{OH}^{-} \ldots \ldots \ldots \ldots . \ldots . \ldots . \ldots 30$

7.1 Partition coeficients into 2-ethyl-1-hexanol for the solutes investigated.................40

7.2 Equilibrium constants of complexation $\left(\mathrm{dm}^{3} \mathrm{~mol}{ }^{-1}\right)$ with borate ion, $\mathrm{B}(\mathrm{OH})_{4}$, in aqueous solution.......47

7.3 Comparison of results for lactic acid extraction by $\mathrm{NPB}^{-}$extractant and $\mathrm{TOMA}^{+} \mathrm{Cl}^{-}$only...........66

7.4 Equilibrium constants of complexation $\left(\mathrm{dm}^{3} \mathrm{~mol}^{-1}\right)$ of solutes with borate in aqueous conditions and NPBin organic conditions.......................69

7.5 Results for sorbitol recovery experiments..........70

A-I Experimental data for glycerol extraction experiments.............................

A-2 Experimental data for fructose extraction experiments........................... 76

A-3 Experimental data for sorbitol extraction 
experiments...........................

A-4 Experimental data for 1,2-propanediol extraction

experiments............................. 78

A-5 Experimental data for 1,2-propanediol extraction using toluene as diluent....................79

A-6 Experimental data for lactic acid extraction

experiments............................ 80 


\section{IIST OF FIGURES}

\section{Figures}

1.1 Molecular structures of solutes investigated........3

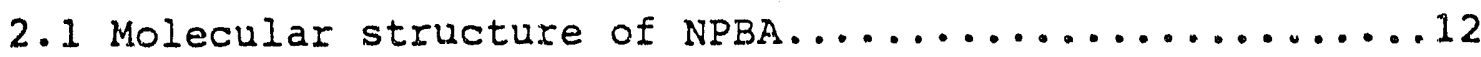

2.2 Possible reaction scheme for the complexation.......12

2.3 The solute in an aqueous phase is "pulled" into the organic phase via complexation with NPB" anion

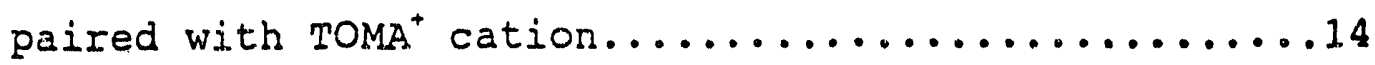

3.1 Batch extraction procedure..................23

4.1 Preparation of the organic extractant...........27

7.1 Glycerol extraction results expressed in the form of a loading curve........................ 42

7.2 Comparison of capacities of two extractants with different degrees of initial treatment by alkali....43

7.3 Experimental loading curve for glycerol compared to theoretical loading curve calculated by using

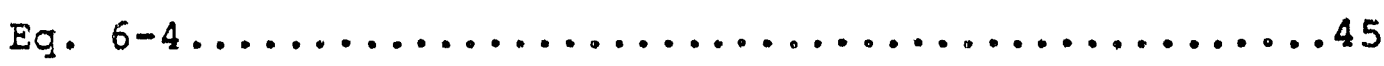

7.4 Glycerol extraction results expressed as distribution ratio (D) plotted against equilibrium concentration of $\mathrm{NPB}^{-}$in the organic phase.................46

7.5 Extraction results for fructose................49

7.6 Experimental loading curve for fxuctose compared to theoretical loading curve calculated by using Eq.6-4................................

7.7 Fructose extraction results expressed as distribution ratio (D) plotted against the equilibrium concentration 


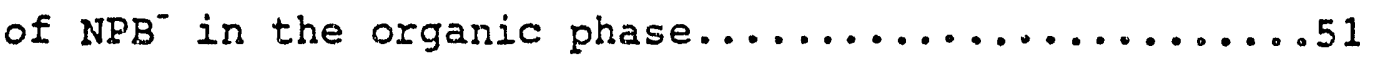

7.8 Extraction results for sorbitol expressed in the

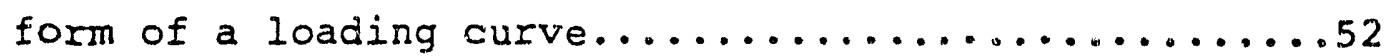

7.9 Sorbitol extraction results expressed as distribution ratio (D) plotted against the equilibrium concentration

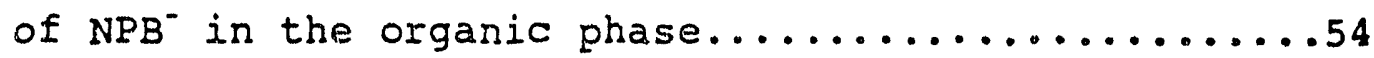

7.10 Experimental loading curve for sorbitol compared to theoretical loading curve calculated by using

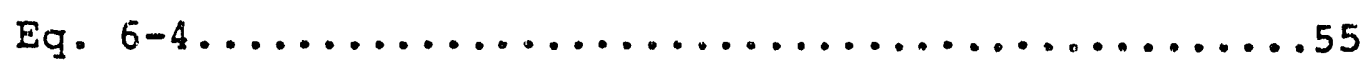

7.11 Fitting theoretical loading to experimental data....56

7.12 Extraction results for 1,2-propanediol expressed in the form of a loading curve.................57

7.13 Comparing results of forward extraction with back extraction for 1,2-propanediol..............60

7.14 Comparing extraction results obtained by using two different diluents...................62

7.15 Iactic acid extraction results expressed in the form of a loading curve..................64

7.16 Lactic acid extraction results after effect of TOMA had been removed..................68 


\section{INTRODUCTION}

Economical recovery of -OH bearing compounds from aqueous solutions is important because they are present in many process streams where either the $-\mathrm{OH}$ bearing compound is the product of interest (e.g., fermentation broths), or there is a need to remove them ( $6 . \mathrm{g}$. , aqueous waste streams). However, these streams are typically dilute in the solute of interest and complex in composition, features which make separation processes challenging and costly. For example, typical fermentation broths contain 0.5-5z of the desired product in a complex aqueous mixture or buffering agents, nutrients, cell debris and other components (Busche,1983), and rypical downstream processing costs for these streams constitute 408 or more of the final product cost (Stowell, 1.986).

Efficient and economical separation methods are needed for these process streams. Such methods should allow direct recovery and selectively remove the desired solute from the complex solution. It is helpful for the separation to remove the solutes of interest, without the need for the large energy expense which would be needed for removal of most or al: of the water. As will be seen below, many of these substanees are presently isolated from rather dilute aqueous soluions by evaporation of the water, an approach which is energyintensive and does not provide the wherewithal for separating among the various solutes which may be present. Further 
complicating matters is the fact that most -OH bearing substances are difficult to solidify.

Our research has focussed upon separation of -OH bearing solutes from dilute aqueous solutions via reversible chemical complexation. The solutes investigated are lactic acid, glycerol, fructose, sorbitol and propylene glycol. Fig. 1.1 shows the molecular structures of these substances.

\subsection{Chemicals from Biomass}

Most commodity chemicals are currently produced from petroleum resources. However, fluctuating petroleum costs, uncertainty of supply and concerns relating to the depletion of these non-renewable fossil fuels mean that we need to develop other resources for chemicals. Biomass is an attractive and abundant alternative. Chemicals most attractive for manufacture from biomass are those which can be made by fermentation, are sufficiently valuable, and have substantial existing or potential markets.

\subsection{Lactic Acid}

One example is lactic acid. About half of the world demand for lactic acid is produced by fermentation (Ward,1989). Lactic acid possesses two functionally active 
Glycerol

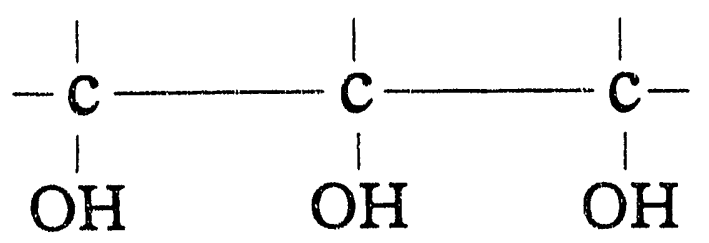

1,2-propanediol

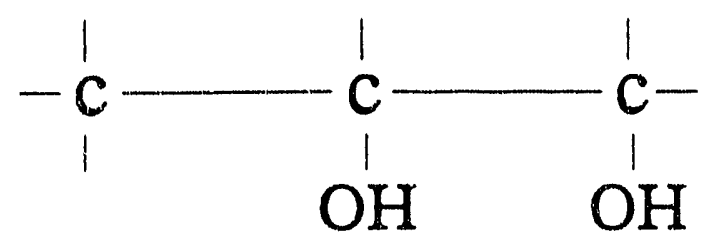

D-Fructose

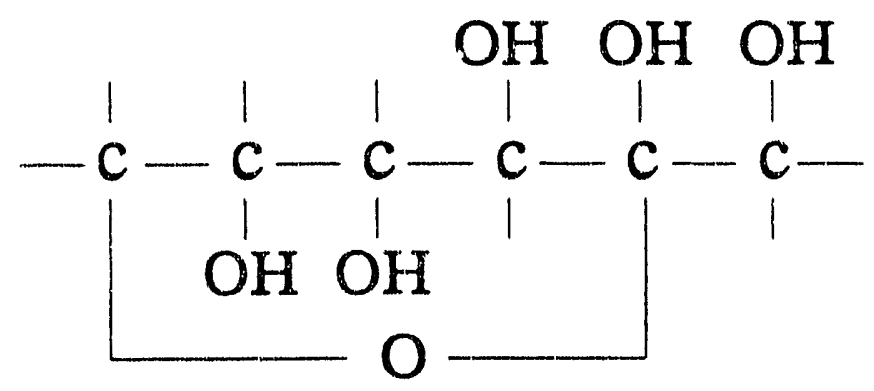

Sorbitol<smiles>CC(C)(O)C(C)(O)C(O)(O)C(C)(O)C(C)(O)C(C)(O)I</smiles>

Lactic acid

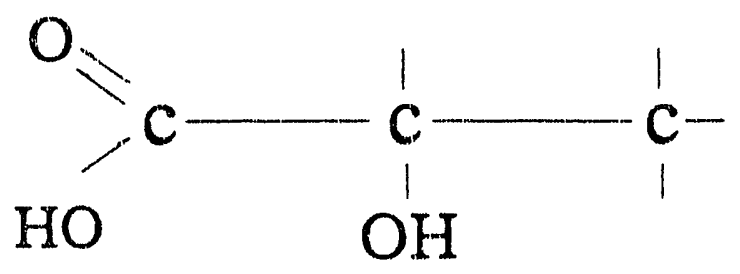

Fig.1.1 Molecular structures of solutes investigated 
groups, $a$-hydroxyl and carboxyl, which make it a potential precursor for numerous value-added chemicals (Ward, 1989). It is also presently receiving much attention as a monomer for production of biodegradable polymers. However, lactic acid is presently relegated to uses in low-volume specialty markets, due to the relatively high costs of production, which are attributable more to recovery and purification than to the fermentation itself (Jain, et al, 1989).

The main industrial uses of lactic acid are as a food acidulant ( 50 of the market), for manufacture of stearoyl-2lactylate $(20 \%)$ and in pharmaceutical and other applications (Ward, 1989). I-(+)-lactic acid is produced by anaerokic fermentation using Lactobacillus debruckii and related homofermentative bacteria (Ward, 1989). Concentrations of up to around $10 \%$ lactic acid can be achieved. The recovery process has been precipitation with calcium carbonate or calcium hydroxide, followed by acidification of the calcium lactate salt by sulfuric acid. The resultant aqueous acid solution (about 108 lactic acid) is concentrated to 508 , refined over carbon, treated with sodium ferrocyanide, and passed through an ion exchange column for final finishing (Ward, 1989).

\subsection{Propylene Glycol}

Propylene glycol (1,2-propanediol) is another example of 
a chemical that can be produced from biomass, although chemical conversion is primarily used. Propylene glycol is used in food and pharmaceutical applications and in unsaturated polyester resins for fiberglass-reinforced products, such as appliances and cars (Chemical Marketing Reporter, February 9,1987).

Racemic propylene glycol is currently produced by hydrolysis of propylene oxide derived from petrochemical sources. The water in the product stream (about 158 propylene glycol) is removed by multiple-effect evaporation, and the product is purified by vacuum distillation (Reiche and Heckman, 1976). R-propylene glycol can be produced by fermentation using the bacterium clostridium thermosaccharolyticum, with glucose as the substrate (Cameron and Cooney, 1986, 1987). Product concentration is about 18 and byproducts such as acetate, Iactate and ethanol are formed.

\subsection{Glycerol}

Glycerol was produced by fermentation for explosives (nitroglycerin) manufacture during world wars $I$ and II. Glycerol is formed by yeast in small amounts along with ethanol in the alcoholic fermentation. Current commercial production of glycerol involves both synthesis from propylene and saponification of glycerides. World production of glycerol is $450-600 \times 10^{3}$ tons/yr, and $f(x$ the past 20 years synthetic 
glycerol has accounted for about one half of the United States annual production (Kirk and Othmer, 1977). Glycerol is used in nearly every industry, with the largest single use being in the marufacture of alkyd resins. It is also used in tobacco processing, drugs, cosmetics and foods.

The product from synthesis from propylene is a dilute aqueous solution containing 58 or less glycerol. This crude glycerol is concentrated to about 808 in multiple-effect evaporators, and salt is removed by centrifuging. Additional concentration of the product, followed by desalting, yields 98 glycerol.

Glycerol from glycerides can be obtained from two sources -- soap manufacture and fat splitting. Both result in aqueous solutions containing about 8 to 208 glycerol. Chemicals such as ferric chloride, caustic soda, calcium chloride, etc., are added to remove impurities. The dilute glycerol liquors, after purification, are concentrated to crude glycerol by evaporation under vacuum, heated by low-pressure steam. The crude glycerol is then refined by distillation, followed by treatment with active carbon.

\subsection{Sorbitol}

Sorbitol belongs to the sugar alcohol family. Annual world production capacity outside the united States is about 159,000 tons, and the production in the united states grew at 
an average rate of 6.38 in the $1965-1974$ period (Kirk and othmer). Sorbitol is used to impart body, texture and sweetness to frozen desserts, as a bodying agent in pharmaceutical syrups and elixirs, as a humectant and emollient.in cosmetics and in textile bleaching or scouring solutions.

Sorbitol is synthesized commercially by high pressure hydrogenation of glucose, usually with a niukel catalyst. Corn syrup is the most important raw material. The sorbitol solution produced is purified by passage through an ionexchange resin bed to remove ions and then treated with activated carbon to remove trace organic impurities. The solution of pure sorbitol is concentrated in a continuous evaporator to a solution containing $70 \%$ solids and sold as is, or else it is further concentrated and crystallized.

\subsection{Fructose}

The most common use of sugar is as a sweetener in the food industry. Fructose is popular as a dietary sugar because it is 1.3-1.8 times as sweet as sucrose or glucose ( $\mathrm{Kim}$, et al. 1985). High fructose content syrups are used by the food industries for producing low calorie foods and drinks (Barker, 1983). Previously fructose was obtained from the hydrolysis of inulin, which was very costly. Nowadays, fructose is produced by separation from the enzymatically isomerized mixture 
containing fructose and glucose, commercialy called high fructose syrup. The isomerized fructose corn syrup typically contains 428 fructose, $50 \%$ glucose, and $8 \%$ oligosaccharides on a dry weight basis (Kim, et al. 1985). The mixture is equivalent in sweetiess to invert sucrose, the 50-50 mixture of D-glucose and D-fiuctose obtained on hydrolysis of sucrose by acid or the enzyme invertase, but with a lower selling price (Barker, 1983). The United States now produces more than $2.3 \times 10^{6}$ metric tons of isomerized syrup annually (Kirk and Othmer, 1977).

In the production of high fructose syrup, corn starch is pasted in a steam jet cooker and then passed over a fixed column of glucoamylase to produce high quality D-glucose. This syrup is passed through a column of fixed isomerase enzyme to give the equlibrium mixture od D-glucose and D-fructose. Either the mixture is sold as a syrup or the D-fructose is concentrated to higher levels by using calcium ion-exchange columns.

In addition to product streams, selective and energy efficient separation methods can also be applied to treatment of aqueous streams to remove fructose commonly found in waste streams from sugar refineries, wineries and distillery. Table 1.1 lists the characteristics of a typical molasses-based distillery spent wash which shows the amount of fructose in the stream and the complexity of the solution. 
TABLE 1.1 Characteristics of Molasses-based distillery system (Karhadkar, et al, 1990)

\begin{tabular}{|c|c|}
\hline Characteristi $: s$ & Average conc. $(\mathrm{g} / 1)$ \\
\hline pH & 4.4 \\
\hline Temperature & $98^{\circ} \mathrm{C}$ \\
\hline Total solids & 97.0 \\
\hline Ash & 52.4 \\
\hline TVA acetic acid & 3.2 \\
\hline Nitrogen & 2.1 \\
\hline Sodium & 2.7 \\
\hline Potassium & 9.5 \\
\hline Calcium & 2.2 \\
\hline Phosphorus & 0.15 \\
\hline Sulphate & 2.5 \\
\hline Glucose & 13.5 \\
\hline Fructose & 12.5 \\
\hline
\end{tabular}

\subsection{Purpose of this project}

This project investigates the possibility of utilizing reversible chemical complexation for removal and recovery of the aforementioned $-\mathrm{OH}$ bearing substances from dilute aqueous solutions. Reversible chemical complexation is one of the few methods capable of direct and selective recovery of the desired solute out of the complex solution. The fact that the complexing agent may react with certain functional groups on solutes can engender high selectivity and uptake capacity. 
Moreover, separation based on chemical complexation typically provides a high capacity at low solute concentration, since for low solute concentrations there is a high driving force of free association sites of the complexing agent.

Most of the solutes considered here are very difficult to separate from aqueous solution by ordinary solvent. extraction. Diols, for example, are very hydrophilic and have Low equlibrium distribution ratios; for ambient conditions no values above 0.7 are reported for propylene glycol (Arenson,1989; Leo, et al, 1971). Lactic acid also has a strong affinity for water due to the presence of a hydroxyl group and a carboxylic acid group. The distribution ratios for common solvents have been reported to range from 0.04 to 0.82 (Short and Eaglesfield, 1952). Therefore, incorporation into the organic phase of an agent that will complex with the solute can potentially increase the economic viability of solvent extraction substantially. 


\section{SELECTION OF EXTRACTANT SYSTEM}

The extractant system used in this study is the same as that used by Randel in a precursor project. A brief explanation of how each component was selected is given below. Please refer to Randel (1991) for a more detailed description.

The complexing agent investigated is 3-nitrophenylboronic acid (NPBA), shown in Fig. 2.1. It possesses adjacent -OH groups that serve to complex reversibly with cis-vicinal hydroxyl groups on the solute. It has been found that complex formation takes place only when the NPBA is in its anionic form (Randel, 1991). Fig.2.2 shows how the complex is thought to be formed. The substituted organic ligand on the boron eliminates the capacity for 1:2 complexes, thereby making it easier to study the complexation reactions. Further, NPBA is characteristic of extractants or complexing sorbents that might be iser in practice.

NPBA is slightly soluble in water $(0.4 \mathrm{wt} / \mathrm{s})$, but very soluble in organic solvents such as alcohols and ethers. Solubility losses could be reduced still further by using an organoboronate with greater aliphatic and/or alkyl weighting. Another reason for the selection of NPBA is that the acid has a $\mathrm{pK}_{\mathrm{a}}$ of 7.1 (Barker,et al, 1973), whereas most other organoboronates have much higher values of $\mathrm{pK}_{\mathrm{a}}$. The electronwithdrawing nitro group stabilizes the anionic complex. This means that complex formation occurs under near-neutral 


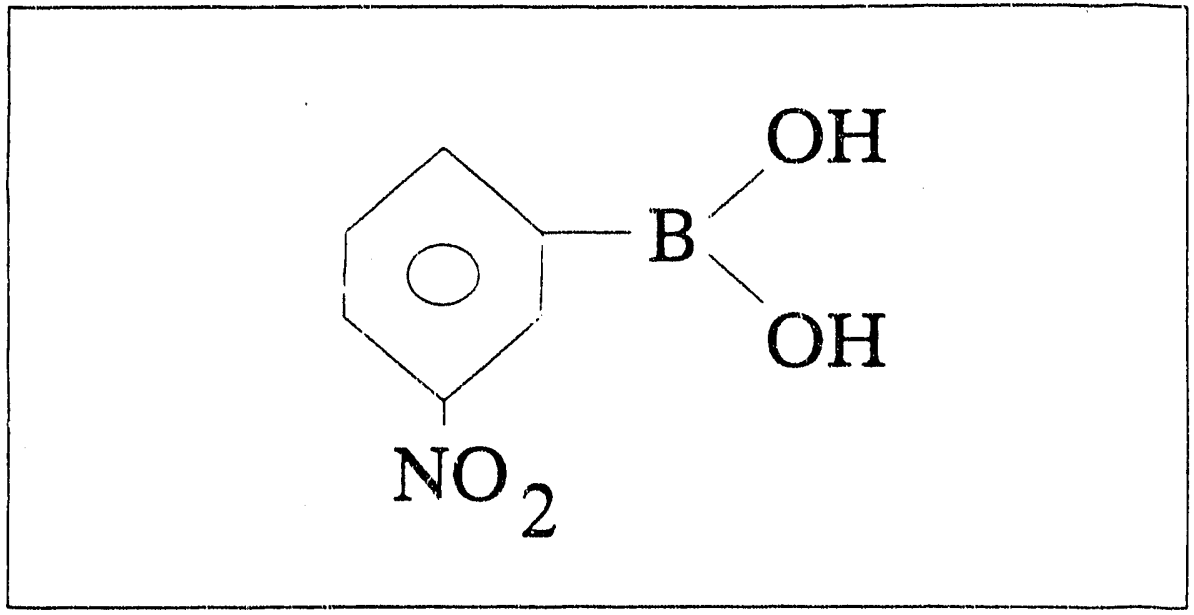

Fig.2.1 Molecular structure of NPBA

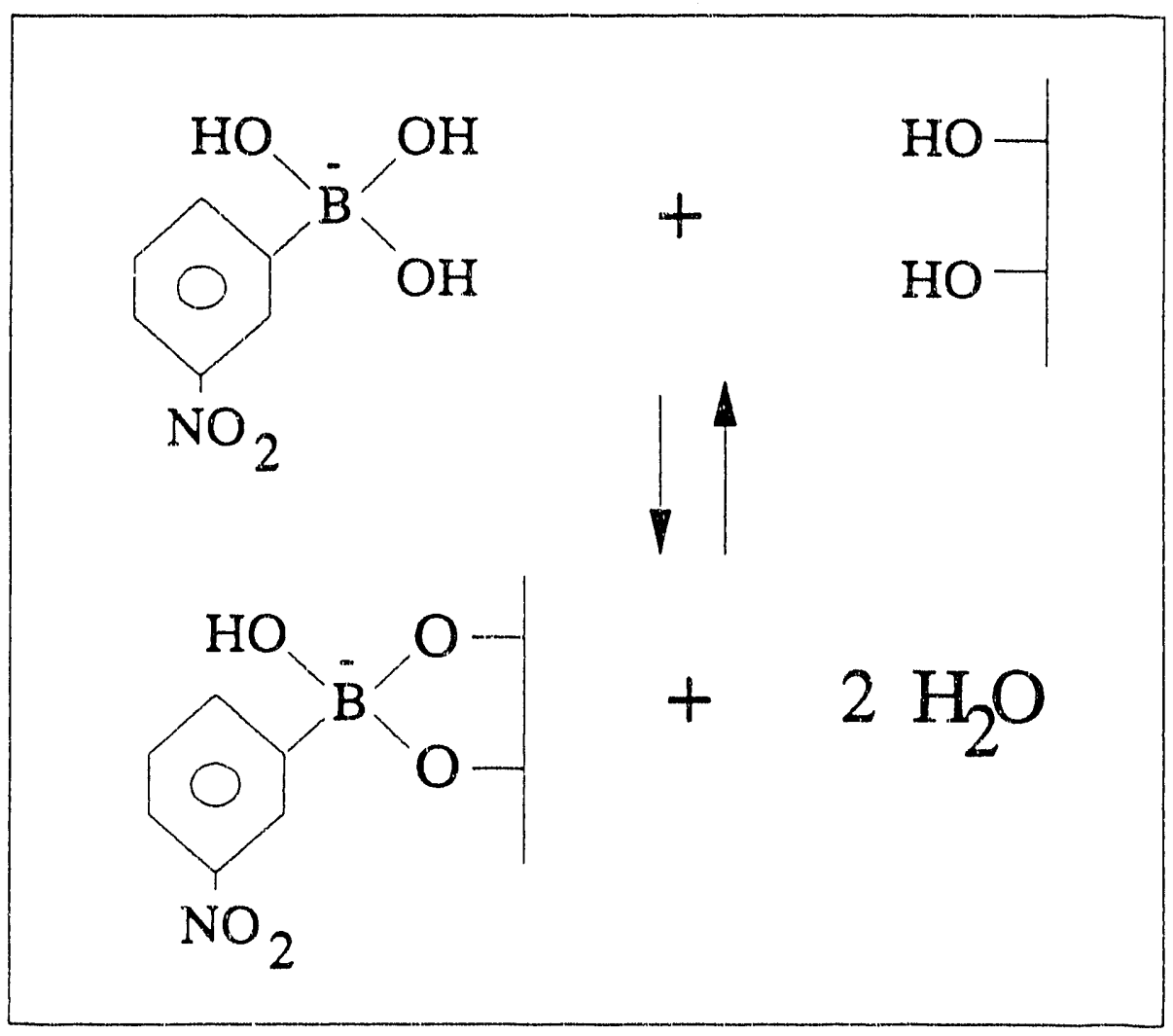

Fig. 2.2 Possible reaction scheme for the complexation 
conditions, which are closer to the conditions of fermentation broths and waste streams. Also, for $\mathrm{pH}$ close to 7.0 , a minimum amount of chemicals will be required for regeneration by $\mathrm{pH}$ swing .

Since the NPBA complexes only in its anionic boronate form ( $\left.\mathrm{NPB}^{-}\right)$, the solvent system for extraction should consist of the organoboronate anion, organic cation, and probably also a diluent, or co-solvent. Aliquat 336 (Aldrich), a quaternary ammonium compound (primarily trioctylmethylammonium ion, TOMA $^{+}$) was used as the organic cation (Fig. 2.3). Mixed ionic extractants have been used previously for extraction of inorganic acids and salts from aqueous solution (see, e.g., Grinstead, et al, 1969; Lynn and Charlesworth, 1972)

A diluent is required for the system because NPBA is a solid. The diluent should be able to dissolve NPBA and Aliquat 336 in sufficient concentration and solvate the TOMA ${ }^{+} \mathrm{NPB}^{-} . \mathrm{S}$ complex. 2-Ethyl-1-hexanol was selected as diluent since it meets all these requirements (Randel, 1991). Toluene was also used as diluent in one set of experiments to determine the effect of a different diluent on the solvation of the complex, as well as to reveal any effect of 2-ethyl-1-hexanol competing with the $-O H$ bearing solutes for binding sites on NPBA anions. 

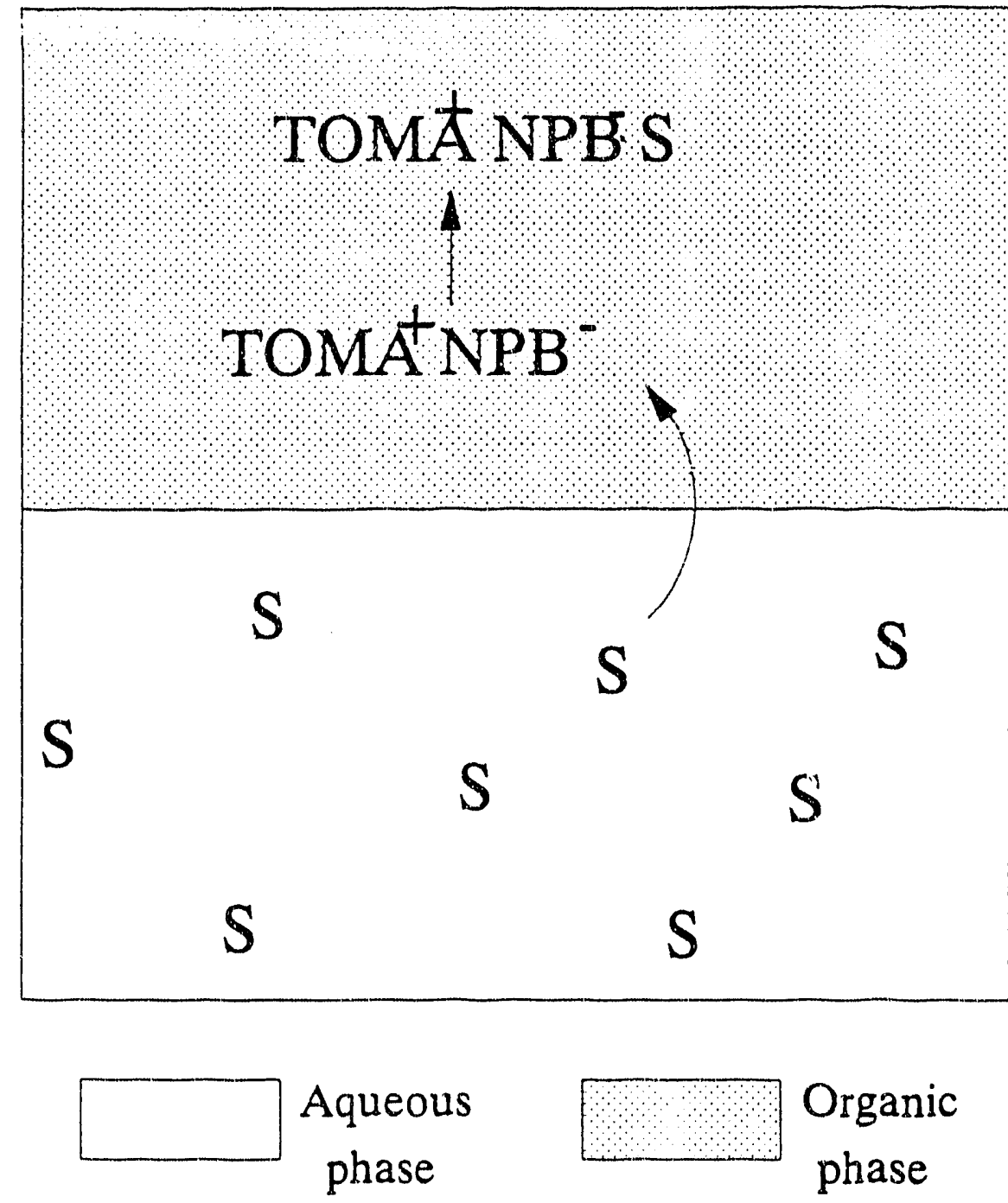

Fig. 2.3 The solute (S) in an aqueous phase is "pulled" into the organic phase via complexation with $\mathrm{NPB}^{-}$anion paired with $\mathrm{TOMA}^{\dagger}$ cation. 


\section{EXPERIMENTAI}

\subsection{Goals}

The purpose of this research was to investigate the feasibility of using the $3-n i t r o p h e n y l b o r o n a t e$ anion as a complexing agent for recovery of compounds with multiple $-O H$ groups from dilute aqueous solution. This study is a continuation of the work of Randel (1991) with propanediols. We ajm to devise a better extracting system with a higher capacity and extend the investigation from propanediols to other solutes of interest, as mentioned in Chapter 1.

Data obtained through batch extraction experiments allowed paraneters such as the distribution ratio (D) and stoichiometric loading ( 2 ) to be calculated and used as means of comparison of complexation behavior amoung various solutes of interest. We also sought to compare the results obtained in this study with aqueous borate complexation data from the literature.

\subsection{Materials}

The chemicals used are listed in Table 3.1. All of them were used as received, without further purification. Aqueous solutions of known concentrations were prepared by dissolving weighed amounts of the solute of interest in 18-megohm 
distilled, deionized water from a Millipore Milli-Q water purification system. Lactic acid was diluted to about $15 \mathrm{w} / \mathrm{w}$ and refluxed for 10 hours at atmospheric pressure to ensure that all dimers and multimers were hydrolyzed to monomers. 2Ethyl-1-hexanol was presaturated with pure distilled water before use. The solubility of water in 2-ethyl-1-hexanol is $2.68 \mathrm{w} / \mathrm{w}$ (Flick, 1985).

TABLE 3.1 Chemicals Used

\begin{tabular}{|l|l|l|}
\hline Chemicals & Purity & Supplier \\
\hline 1,2-Propanediol & $99 \%$ & Aldrich \\
\hline Glycerol & 998 & Mallinkrodt \\
\hline Fructose & - & Eastman Organic \\
\hline Sorbitol & - & Eastman Organic \\
\hline Lactic Acid & reagent grade & Mallinkrodt \\
\hline $\begin{array}{l}\text { 3-Nitrophenyl- } \\
\text { boronic acid (NPBA) }\end{array}$ & & Aldrich \\
\hline Aliquat 336 & & Aldrich \\
\hline 2-Ethyl-hexanol & 998 & Aldrich \\
\hline Sulphuric acid & reagent grade & Mallinkrodt \\
\hline
\end{tabular}


Standardized $0.1 \mathrm{~N}$ and $0.01 \mathrm{~N} \mathrm{HCl}$ and $\mathrm{NaOH}$ solutions were used for acid-base titrations. Standardized $\mathrm{Cl}^{-}$solutions (NaCl) were used for $\mathrm{Cl}^{-}$concentration measurements. Sulfuric acid was diluted to the desired concentration (0.01N) and filtered with Milipore type-HA 0.45 micron filters before use. Compressed gases were obtained in cylinders from the University of California, Berkeley, College of Chemistry.

\subsection{Purity of materials}

Aliquat 336 (primarily trioctylmethylammouium chloride, TOMA ${ }^{+} \mathrm{Cl}^{\prime \prime}$ ) is a quarternary ammonium compound supplied in the cl-form. The manufacturer reports that $C_{8}$ chains predominate in Aliquat 336 and that the formula weight is 404.17 . However, an elemental analysis done by the UCB Microanalytical laboratory (Table 3.2) indicites that more carbon was present than expected, ind chloride concentration was measured to be $6.638 \mathrm{w} / \mathrm{w}$, compared to $8.778 \mathrm{based}$ on the formula weight. The disagreement may be explained by a predominance of $c_{20}$ chains rather than $C_{\beta}$ chains. Alternatively, there may be a significant alkane-like impurity. Moreover, the o wt/wt for the elements in Table 3.2 adds up to about $88 \%$; adding to this the $C I^{\circ}$ concentration $(6.63 \%)$ increases the sum to about $95 \%$. The "missing" 58 could be due to analytical error, or some other elements might be present. One possible additional elenent might be oxygen in the form of hydroxide ions, acting 
as counterions for the TOMA ${ }^{+}$in place of chloride ions. The chloride concentration of $6.63 \% \mathrm{w} / \mathrm{w}$ was used in subsequent calculations.

The manufacturer reports the formula weight for the NPBA as 166.93, but Randel (1991) determined the formula weight to be 148.91 , which is the formula weight of the corresponding anhydride, which has the same melting point as that of the NPBA, as reported by the manufacturer.

TABLE 3.2 Analysis of Aliquat 336

\begin{tabular}{|l|l|l|}
\hline Element & wt/wt & mols/100g \\
\hline Carbon & 71.31 & 5.94 \\
\hline Eydrogen & 13.65 & 13.54 \\
\hline Nitrogen & 2.99 & 0.21 \\
\hline
\end{tabular}

* Ratio of $C: N=5.937 / 0.2131=27.85$. If all chains were $C_{8}, C: N=25$. This indicate that some side chains contain more than eight carbons. 


\subsection{Chemical Analyses}

\subsubsection{Elemental analyses}

Chloride : Aqueous samples were analyzed in the laboratory with an Orion Model 96-17B combination chloride electrode.

Boron : Samples were sent to the UCB Microlab for analysis by flame atomic absorption spectrometry.

\subsubsection{Water}

Water concentrations in the organic extractant were determined with a Quintel Computrac MS-1 Karl Fisher Titrator, using the $2 \mathrm{mg} \mathrm{H}_{2} \mathrm{O} / \mathrm{ml}$ titer Karl Fischer Reagent from Ericson Instruments.

\section{4 .3 Solutes in agueous solutions}

The solutes investigated were 1,2-propanediol, glycexol, fructose, sorbitol and lactic acid. The concentrations of the solutes in aqueous solutions were determined by HPLC (High Performance Iiquid Chromatography). The systom consisted of a Perkin Elmer Series 10 Liquid Chromatography pump with a $20 \mu l$ Rheodyne injection loop, a Bio-Rad Fast Acid column (packing: sulphonated divinylbenzene-styrene copolymer, 9 
micron, 8\% crosslinked; mechanism: reverse phase partitioning onto hydrophobic backbone) and a Waters model 401 refractive index detector. The samples were analyzed at $60^{\circ} \mathrm{C}$ with a mobile phase of $0.01 \mathrm{~N} \mathrm{H}_{2} \mathrm{SO}_{4}$ at $0.85 \mathrm{ml} / \mathrm{min}$. Lactic acid solutions were also analyzed by titration with a Ross model 8103 combination $\mathrm{pH}$ electrode. Titrant solutions of $0.01 \mathrm{~N}$ and $0.001 \mathrm{~N}$ NaOH were prepared by dilution of standardized $0.1 \mathrm{~N}$ NaOH solutions.

\section{4 .4 NPBA}

The NPBA concentrations in aqueous extraction samples were determined by HPLC with the same system mentioned above, except that a Waters Model 440 Fixed Wavelength (254nm) UV detector was used instead of the RI detector.

The NPBA concentrations in the aqueous wash solutions during organic phase treatment (Section 3.5) were determined by boron analyses.

\subsection{Procedure}

\subsubsection{Preparation of the Organic Extractant}

The components of the orgaric phase are listed in Table 3.3. NPBA and Aliquat 336 in approximately equal molar amounts were measured into volumetric flasks, and diluent (2-ethyl-1- 
hexanol or toluenel was added to the desired volume to dissolve the NPBA and Aliquat 336. The organic solutions were then washed with one or more equal volumes of NaOH solutions, having the same molar concentration as the NPBA. The purpose of this was to convert the NPBA to its anionic form, since previous work has indicated that NPBA must be in its anionic form to complex.

TABLE 3.3 Components of Organic Extractant

\begin{tabular}{|c|c|c|}
\hline Components & F.W. & Specific Gravity \\
\hline 2-Ethyl-hexanol & 130.22 & 0.8339 \\
NPBA & 148.91 & (solid) \\
Aliquat 336 & 500.00 & 0.884 \\
\hline
\end{tabular}

Several batches of these organic extractants were made throughout the work. All except the first batch were washed twice with $\mathrm{NaOH}$ solution, followed by a final wash with an equal volume of pure distilled water. The first batch was washed five times with successive batches of $\mathrm{NaOH}$ solution, and showed a subsequent decrease in the loading capacity, as will be discusizd in Chapter 7. The aqueous alkali wash 
solutions after the treatment were analyzed for boron and chloride concentrations so as to enable the final anionic NPBconcentration in the organic phase to be inferred. The goal was to obtain close to $100 \%$ ionization.

Another approach for treating the organic phase is to dissolve Aliquat 336 in the diluent first and then mix this solution with the $\mathrm{NaOH}$ solution to convert $\mathrm{TOMA}^{+} \mathrm{Cl}^{-}$to TOMA ${ }^{+} \mathrm{OH}^{-}$, before NPBA is added. An experiment was done in this way and a comparison of results are shown in section 5.2 .

\subsubsection{Eorward Extraction}

Batch experiments were performed for each solute separately (Fig. 3.1). Equal volumes $(5 \mathrm{ml})$ of aqueous solution and organic extractant solution of known concentrations were mixed in $20 \mathrm{ml}$ vials and were sealed with foil at the top so as to minimize evaporation. The vials were equilibrated in a cemperature-controlled shaker bath at $25^{\circ} \mathrm{C}$ for at least 15 hours, which preliminary tests demonstrated to be a sufficient time for equilibration. The aqueous and organic phases were then separated, and the aqueous phase was diluted and filtered before being analyzed by HPLC to determine how much of the solute remained. Mass-balance calculations indicated how much solute was extracted into the organic phase, and other useful parameters were then inferred as described in Chapter 4. Partition coefficients were 

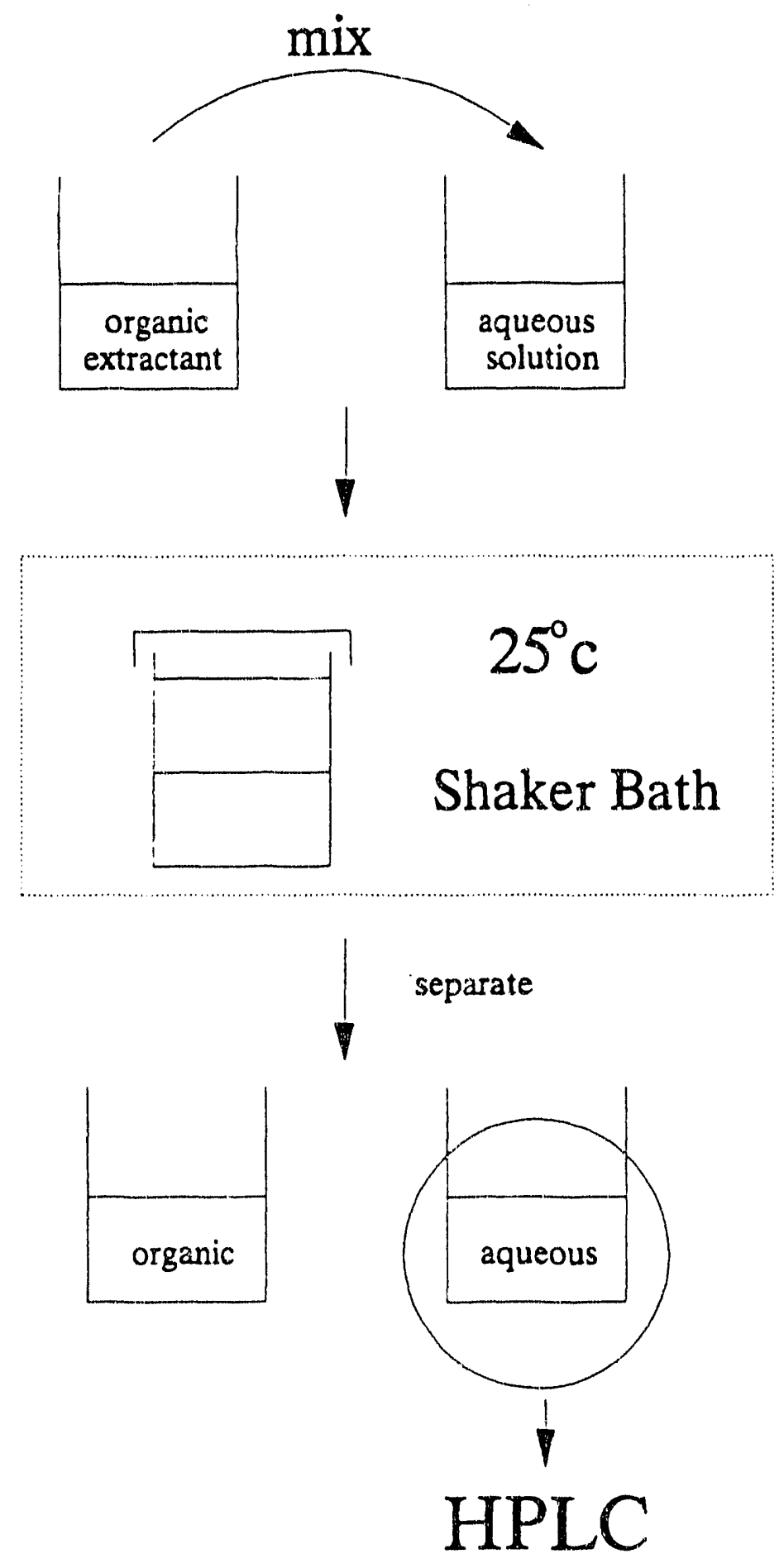

Fig. 3.1 Batch Extraction Procedure 
obtained by contacting the aqueous phase with the diluent only instead of with the extractant mixture.

\section{5 .3 Back Extraction}

Back extraction experiments were carried out with 1,2propanediol to assess whether the forward extraction had attained equilibrium before separation of the phases. The organic phase obtained after forward extraction of 1,2propanediol was mixed with distilled water and equilibrated in the shakex bath again for about 15 hours. The aqueous phase after separation was analyzed in the same way as for the forward extraction.

\subsubsection{Effect of Diluent}

Most experiments were done with 2-ethyl-1-hexanol as the diluent. To check the effect of the polarity and the $-\mathrm{OH}$ group of the diluent on the complexation, a less polar diluent, toluene, was used for some experiments with 1,2propanediol, and the comparison is shown in section 6.2.3.

\section{5 .5 Extraction by TOMA ${ }^{+}$alone}

The same procedure as described in section 3.5 .2 was used, except that no NPBA was added to the organic extractant 
and only the Aliquat 336 was used as extractant. This was done to check whether and to what extent $\mathrm{TOMA}^{+}$itself contributes to the extraction. It has been reported that TOMA+ extracts phenols effectively (Katsutoshi, et al, 1984). 


\section{TREATMENT OF THE ORGANIC EXTRACTANT}

As mentioned in Section 3.5.1, the organic extractant was washed with aqueous $\mathrm{NaOH}$ solutions to ionize the NPBA to NPB-. The wash solutions were then analyzed for $\mathrm{Cl}^{-}$and boron concentration to infer the final $\mathrm{NPB}^{-}$concentration of the extractant. Table 4.1 shows the results of these analyses for one typical treatment.

\section{TABLE 4.1 Results of boron and $\mathrm{Cl}^{-}$analysis of the wash solutions for one typical batch of extractant}

\begin{tabular}{|c|c|c|}
\hline & {$\left[\mathrm{Cl}^{-}\right](\mathrm{M})$} & {$[\mathrm{B}](\mathrm{M})$} \\
\hline 1st NaOH wash & 0.102000 & 0.0625 \\
\hline 2nd NaOH wash & 0.021420 & 0.0289 \\
\hline water wash & 0.000306 & 0.0093 \\
\hline
\end{tabular}

Fig. 4.1 part a) shows a possible mechanism for the ionization and displacement of $\mathrm{Cl}^{-}$: The $\mathrm{OH}^{-}$ions from the wash solution enter the organic phase and ionize the NPBA molecules to $\mathrm{NPB}^{-}$anion. Once an $\mathrm{NPB}^{-}$anion is formed, it pairs with a TOMA cation and a $\mathrm{Cl}^{-}$ion is displaced into the aqueous wash 
a)

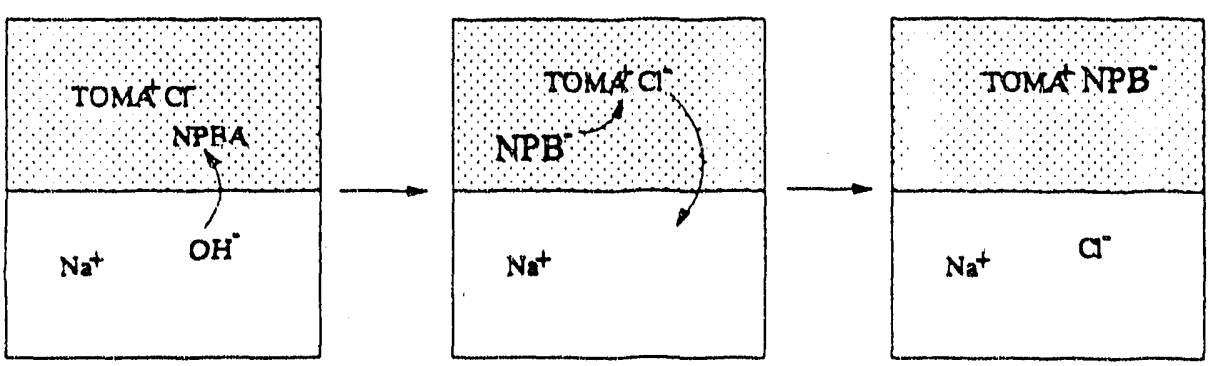

b)

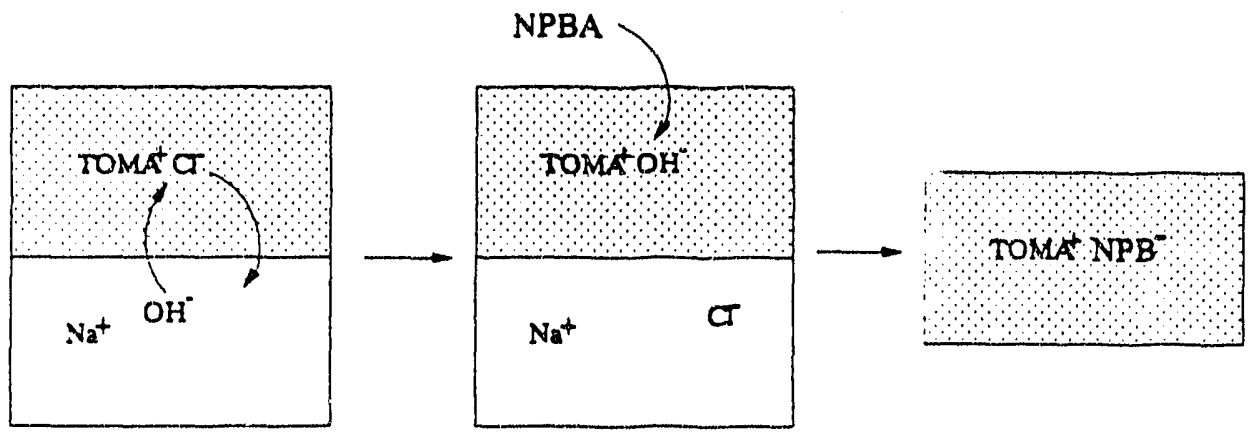

Fig. 4.1 Preparation of the Organic Extractant 
solution, because of the requirement for electroneutrality. It is assumed that $\operatorname{TOMA}^{+}$would not partition into the aqueous phase due to its hydrophobic nature. Therefore, it was assumed that one $\mathrm{Cl}^{-}$ion displaced corresponded to one NPBA molecule ionized to $\mathrm{NPB}^{-}$anion.

\section{I Sample calculation}

Using the data from Table 4.1 above, the concentration of $\mathrm{NPB}^{-}$ions in the extractant was calculated as follows:

* initial concentration of $\mathrm{NPBA}=0.2105 \mathrm{M}$

* initial concentration of $\mathrm{TOMA}^{+} \mathrm{Cl}^{-}=0.1904 \mathrm{M}$

* sum of $\mathrm{Cl}^{-}$concentrations of all wash solutions $=(0.102+0.02142+0.000306) \mathrm{M}$

$=0.12372 \mathrm{M}$

* sum of boron concentrations of all wash solutions $=(0.0625+0.0289+0.0093) \mathrm{M}$

$=0.1007 \mathrm{M}$

* amount of NPBA left in the organic phase $=(0.2105-0.1007) \mathrm{M}=0.1098 \mathrm{M}$

Concentrations were used directly without multiplication by 
volume because the volumes of each wash solution and organic extractant solution were the same.

since the amount of $\mathrm{Cl}^{\circ}$ ions displaced $(0.12372 \mathrm{M})$ is greater than amount of NPBA left in the extractant after washing $(0.1098 \mathrm{M})$, it was assumed that all NPBA was ionized and that the final NPB concentration of the extractant was $0.1098 \mathrm{M}$.

The fact that the amount of $\mathrm{Cl}^{-}$removed was greater than the amount of NPBA remaining suggests that the excess TOMA+ that was not paired with $\mathrm{NPB}^{-}$unight be paired with $\mathrm{Cl}^{-}$or $\mathrm{OH}^{-}$. However, since the amount of NPBA initially present in the extractant was estimated by calculation based on the weight and the estimated molecular weight, there could also be error involved in that estimation.

\subsection{Converting TOKA+CI- to TOMA+OH- first}

Arother approach for treating the extractant is to convert the TOMA ${ }^{*} \mathrm{Cl}^{-}$disscilved in the diluent to $\mathrm{TOMA}^{+} \mathrm{OH}^{-}$ before aduing in the NPBA, as shown in Fig. 4.1, part b. The results of $\mathrm{Cl}^{-}$analysis $\mathrm{O}$ the wash solutions from treating an organic phase in this way are shown in table 4.2 below.

When compared to the results shown in rable 4.1 , it is obvious that the rate of $\mathrm{Cl}^{*}$ displacement is slow using the second method, and nore alkali is needed. Therefore, the first method was used for all extractant treatment. 


\subsection{NPBA losses}

It can be seen from the data in Table 4.1 that the anount of NPBA lost into the aqueous wash solution during treatment is rather large, since NPBA is more soluble in basic than neutral aqueous solutions. This is probably due to the formation of $\mathrm{NPB}^{-}$, which is more easily dissolved into water than is NPBA. This result indicates that losses of the extractant would increase sharply with increasing $\mathrm{pH}$ of the aqueous feed to an extraction using $\mathrm{NPB}^{-}$as the complexant.

TABLE 4.2 PE and $C 1^{-}$analysis of wash solutions for converting TOMA+C1- tO TOMA+OH-

Initial concentration of TOMA $^{+} \mathrm{CI}^{-}=0.1802 \mathrm{M}$

\begin{tabular}{|c|c|c|}
\hline & $\mathrm{pH}$ & {$\left[\mathrm{Cl}{ }^{-}\right](\mathrm{M})$} \\
\hline Ist NaOH wash & 13.04 & 0.03128 \\
\hline 2nd NaOH wash & 13.07 & 0.01104 \\
\hline 3rd NaOH wash & 13.08 & 0.01163 \\
\hline 4th NaOH wash & 13.09 & 0.01010 \\
\hline 5 th NaOH wash & 13.11 & 0.00592 \\
\hline
\end{tabular}




\section{NUMERICAI TREATMENT OF DATA}

In order to make use of the data obtajned experimentally, some numerical manipulation was done to obtain useful parameters such as partition coefficient (P), distribution ratio (D), degree of loading (2),..., etc., which are useful for evaluating process feasibility.

\subsection{Experimentally measured quantities}

The experimental data available for each extraction sample are:

i) (S): the initial concentration of the solute in the aqueous phase $(\operatorname{mol} / I)$.

ii) (S) , the final concentration of the solute in the aqueous phase $(\operatorname{mol} / L)$.

iii) $[\mathrm{N}]_{i}$, the total concentration of $\mathrm{NPB}^{-}$anions in the organic extractant (mol/L).

Parentheses denote aqueous phase concentrations, and brackets denote organic phase concentrations. i.) and ii) were obtained by HPIC; $i i i)$ was obtained as described in section 4.1, adjusted for the degree of dilution.

5.2 Amount of solute extracted into the organic phase

The difference between $(S)_{2}$ and $(S)_{\leq},(S)_{1}-(S)$, was taken 
as the concentration of solute extracted into the organic phase [S] $]_{0, f}$. An assumption is made here that the phase volumes remain equal after extraction.

\subsection{Correction for water comextraction}

The organic phases of the extraction samples were analyzed for water concentration by Karl Fischer titration before and after the extraction. This enabled a determination of whether the effect of water co-extraction on the final phase volumes was significant, i.e., whether the change of phase volumes due to water co-extraction would affect the calculation of the anount of solute in the organic phase.

It was found that the difference in water concentrations in the organic extractant before and after the extraction was typically $\pm 0.38 \mathrm{wt} / \mathrm{wt}$, which is small and was taken to be negligible. This favorable result was facilitated by the presaturation of the 2-ethyl-1-hexanol with water before making up the extractant mixture.

\subsection{Distribution ratio, (D)}

Solvent extraction equilibria are generally reported as distribution ratio, $D$, which reflects the capacity of the solvent for extraction of the solute from the aqueous phase into the organic phase. $D$ is defined in terms of the 
experimentally measured equilibrium concentrations of the solute in the aqueous and organic phases:

$$
D=\frac{C_{\text {org }}}{C_{\text {ag }}}
$$

$C_{\text {org }}=$ molar concentration of solute in the organic phase $C_{a q}=$ molar concentration of solute in the aqueous phase

In terms of the extractant system used here:

$$
D=\frac{[N \cdot S]_{\text {org }}+[S]_{\text {org }}}{(S)_{\text {aq }}}
$$

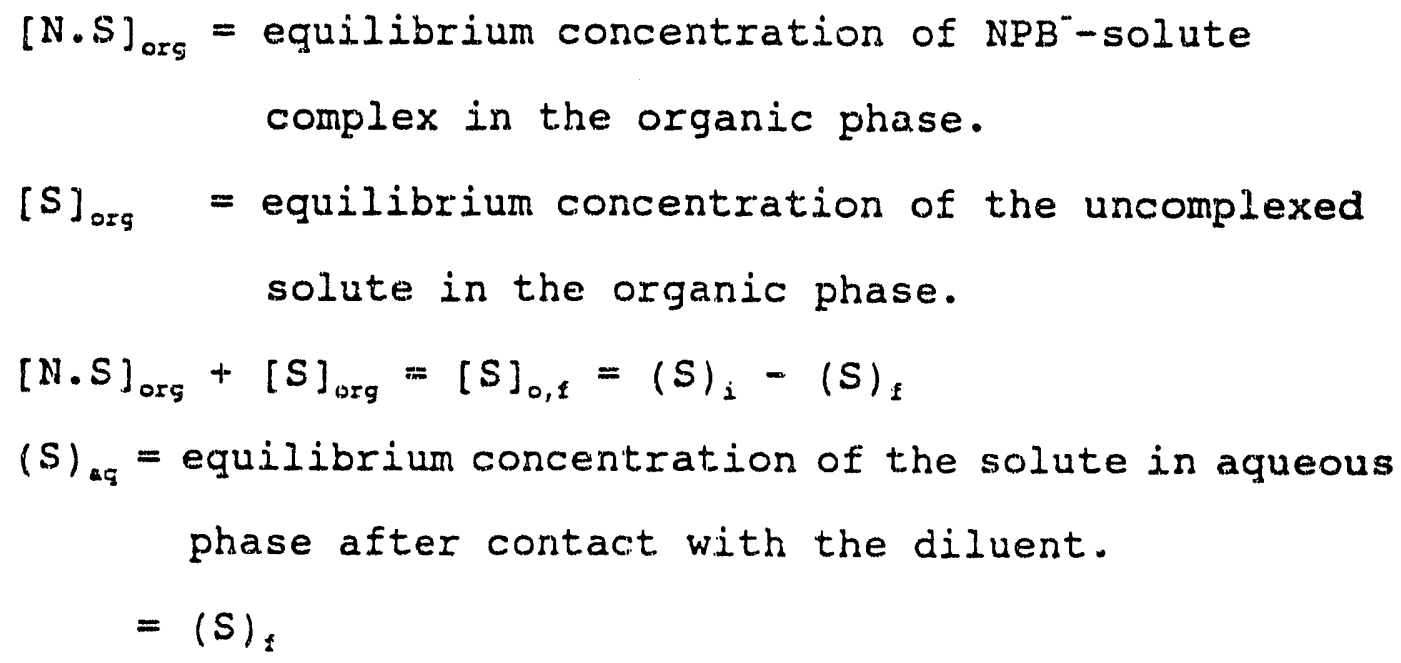

$D$ varies with the concentration of $\mathrm{NPB}-$ in the organic phase and the concentration of solute in the aqueous phase. 


\subsection{Partition coefficient, (P)}

When the organic phase is composed of the diluent only, $D$ becomes $P$, the partition coefficient, under the assumption that there is no complexation of the solute with either itself or the diluent. $P$ provides information on the degree of extraction of solute by the diluent alone and depends upon the natures of the solute and diluent. This information is needed to distinguish the chemical extraction by the complexing agent (NPB-) from the physical extraction by the diluent alone, as discussed below in Section 5.6. P is defined as

$$
P=\frac{[S]_{\text {org }}}{(S)_{a q}}
$$

The values of $P$ for the solutes investigated using 2ethyl-1-hexanol as the diluent are listed in Table 7.1 in Chapter 7 .

\subsection{Correction for Extraction by diluent}

Physical extraction by the diluent alone was taken into account by subtracting a correction factor from the

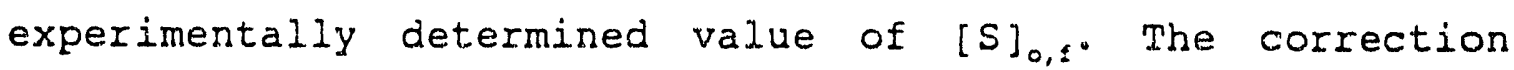
factor at each value of [S], was the concentration of solute extracted by the diluent alone lobtained by separate experiments), multiplied by the volume fraction diluent in the 
solvent mixture:

$$
\text { corrected }[S]_{0, f}=[S]_{0, f}-P \times[S]_{a q} \times V_{f}
$$

$v_{1}=$ volume fraction diluent in the organic phase, which was typically large, above $97 \%$.

The corrected [S] $]_{0, f}$ corresponds to the amount of extraction by the complexing agent (NPB') alone. It is needed for calculating the degree of loading of the extractant, as described below.

\subsection{Degree of loading, (z)}

The stoichiometric loading of the extractant, $z$, is the number of molecules of solute that are complexed per NPB" anion. $z$ is defined as the equilibrium molar ratio of the solute extracted by the extractant to the total extractant in the organic phase :

$$
z=\frac{[N \cdot S]_{\text {org }}}{[N]_{i}}
$$

$[\mathrm{N}]_{i}=$ concentration of $\mathrm{NPB}^{-}$anion in the extractant. 
stoichiometry of the complex. 


\section{CHEMICAI MODELING OF EXTRACTION DATA}

An equilibrium description of the complexation system can be written as :

$$
\mathrm{nN}+\mathrm{mS}-\mathrm{N}_{\mathrm{n}} \cdot \mathrm{S}_{\mathrm{m}}
$$

where $\mathrm{n}$ molecules of NPB" anions, $\mathrm{N}$, are complexed with $\mathrm{m}$ molecules of solute, $S$, to form a complex $N_{n} \cdot S_{m}$.

The relationship can be expressed in terms of a heterogeneous equilibrium constant, $\beta_{\mathrm{nm}}$ :

$$
\beta_{n, a}=\frac{\left[N_{n} \cdot S_{m}\right]_{\text {org }}}{[N]_{o r g}^{n}(S)_{a q}^{m}}
$$

Theoretically, activities of the species should be used, but for practicality the activities of the species are assumed to be proportional to the concentrations, and the constants of proportionality are taken up in the equilibrium constant. For a $1: 1$ complex, the expression would be:

$$
\beta_{:,:}=\frac{[N . S]_{\text {org }}}{[N]_{\text {org }}(S)_{\alpha q}}
$$

From the definitions for distribution ratio and partition coefficient in Chapter 4 : 


$$
\begin{gathered}
D=\frac{[N \cdot S]_{\text {org }}+[S]_{\text {org }}}{(S)_{\mathrm{aq}}} \\
P=\frac{[S]_{\text {org }}}{(S)_{\text {aq }}}
\end{gathered}
$$

From Eq.6-2,

$$
\begin{aligned}
& \beta_{1,2}[N]_{\text {org }}=\frac{[N \cdot S]_{\text {org }}}{(S)_{\text {ag }}} \\
& =D-P \times V_{t} \\
& D=\beta_{1,1}[N]_{\text {org }}+P \times V_{f}
\end{aligned}
$$

Theoretically, by knowing $D,[N]_{\text {org }}$ and $P$ from the experimentally obtained data, values of $\beta_{1,2}$ can be obtained graphically.

The loading of the extractant, $z$, as defined in section 5.7, could be expressed as follows for a $1: 1$ complex (Tamada, 1989) which is derivable from Eq.6-2:

$$
z=\frac{\beta_{1,2}(S)_{\mathrm{Aq}}}{1+\beta_{2,2}(S)_{\mathrm{aq}}}
$$


This equation suggests that loading increases rapidly with increasing (S) as in proportion to $\beta_{1,1}$ at low solute concentration and asymptoticaliy approaches unity at high (S) aq, as the available extractant is exhausted. The experimental extraction results for the various solutes are presented as loading curves [plots of $Z$ vs $\log (S)_{\text {ag }}$ ], which are shown in the following chapter. The theoretical expression of $\mathrm{z}(\mathrm{Eq} \cdot 6-4)$ was also used to "calculate" the theoretical loading curve and was compared to the corresponding experimental curve to test the degree of agreement of experimental data with theory for some of the solutes investigated. 


\section{RESULTS AND DISCUSSION}

\subsection{Partition Coefficients and Distibution Ratios}

The values of $P$ measured for the solutes investigated using 2-ethyl-1-hexanol as the diluent are listed in Table 7.1

TABLE 7.1 Partition coefficients into 2-ethy1-1-hexanol for the solutes Investigated

\begin{tabular}{|c|c|}
\hline Solutes & (P) \\
\hline 1,2 -propanediol & 0.010 \\
\hline glycerol & 0.013 \\
\hline fructose & 0.033 \\
\hline sorbitol & 0.035 \\
\hline lactic acid & 0.078 \\
\hline
\end{tabular}

The fact that $P$ increases as the number of $-O H$ groups increases on the solute suggests that there is, in fact, some complexation or preferential solvation between the solute and 2-ethyl-1-hexanol.

The values of distribution ratios for the various 
experimental measurements are listed in the Appendix.

\subsection{Glycerol}

The experimental results for glycerol extraction are presented in the form of a loading curve in Fig.7.1 (loading plotted against the logarithm of the equlibrium aqueous concentration of solute). The different symbols correspond to different concentrations of $\mathrm{NPB}^{-}$used during extraction. It can be seen that the points tend to group into a single curve instead of forming different curves for different total NPB' concentrations. The fact that there is no apparent dependence of loading on total $\mathrm{NPB}^{-}$concentration suggests that there is only one molecule of $\mathrm{NPB}^{-}$per complex, since the loading would increase with increasing NPB" concentration if there were more than one $\mathrm{NPB}^{-}$per complex (Tamada, 1989).

Fig. 7.2 shows the loading curve measured for glycerol extraction by the first batch of extractant, which was washed five times with $\mathrm{NaOH}$ solution as described in section 3.5.1, compared to the loading curve for extractant washed only twice (Fig. 7.1). It is obvious that extractant washed twice had a higher capacity than extractant washed more extensively. It may be that excessive washing causes greater NPBA losses than were calculated from the aqueous boron analyses. It was shown in Chapter 5 that two consecutive washes appear to be enough to ionize the NPBA in the organic phase nearly completely, and 


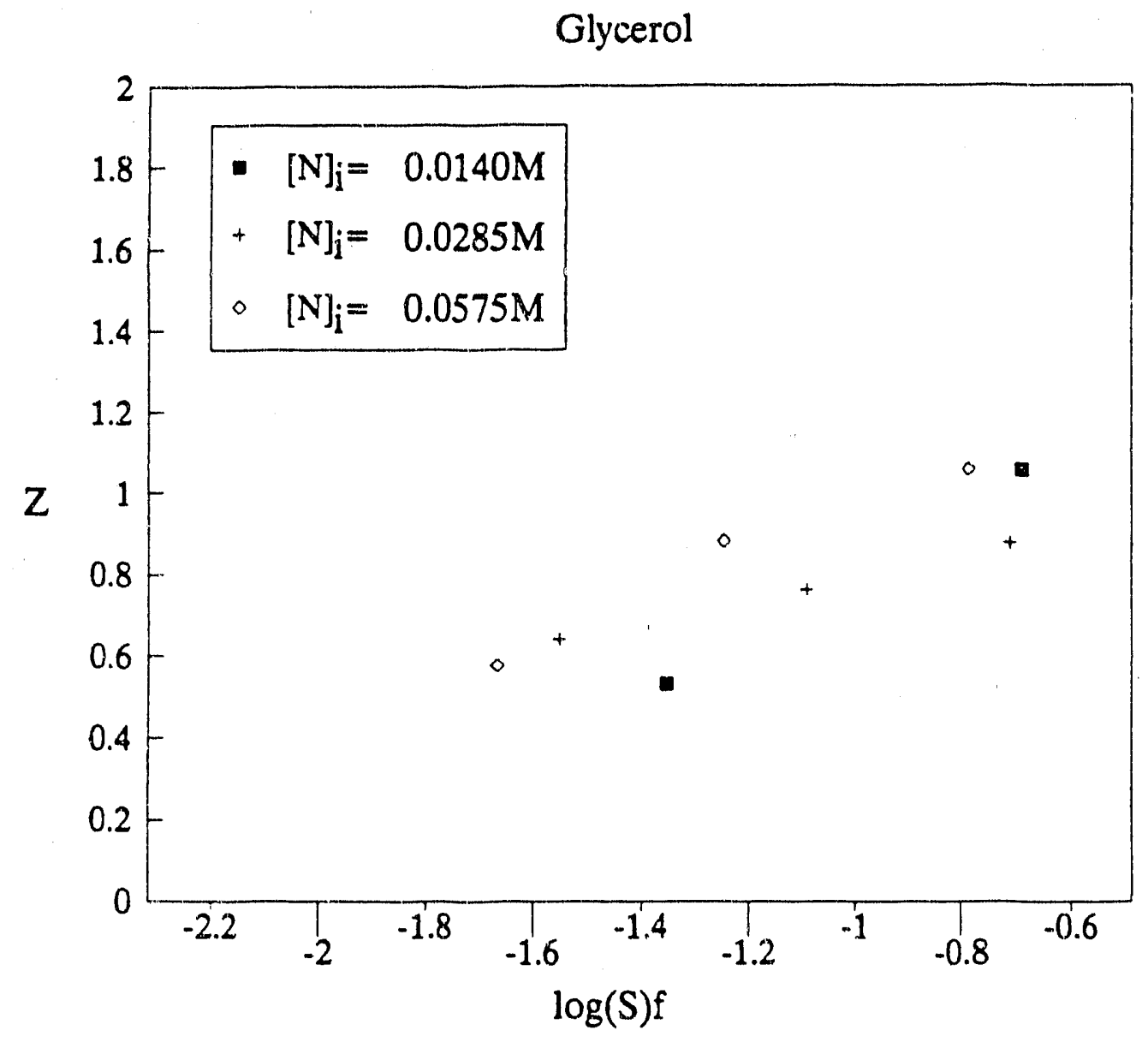

Fig. 7.1 Glycerol extraction results expressed in the form of a loading curve $\mathrm{Z}=$ loading

$(S)_{\mathrm{f}}=$ aqueous solute concentration at equlibrium $[N]_{i}=$ total NPB - concentration in the organic extractant during extraction 


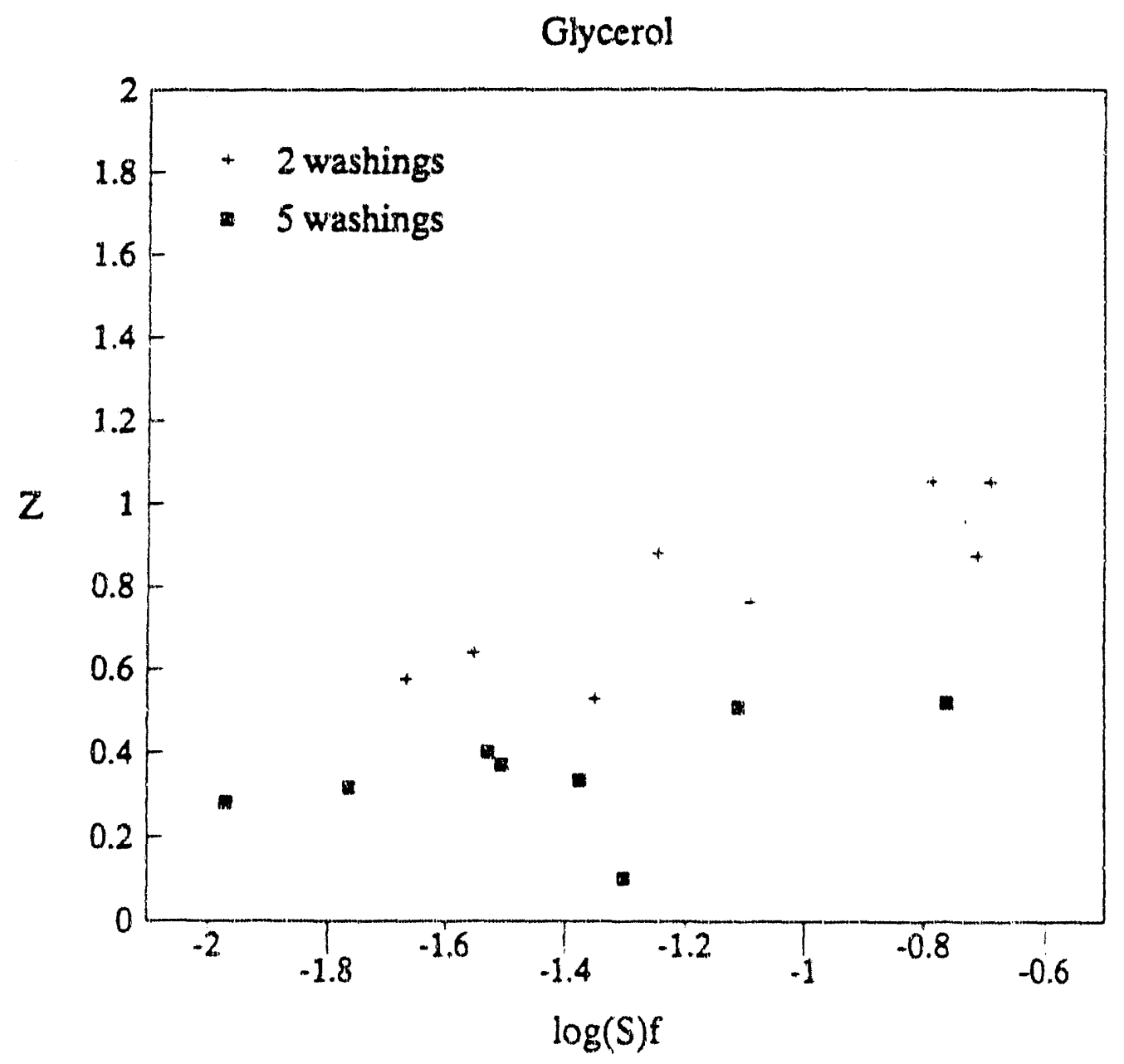

Fig. 7.2 Comparison of capacities of two extractants with different degrees of initial treatment by alkali. 
this was therefore the procedure adopted for all the extractants used in the remainder of this work.

Since there is no overloading apparent from Figure 7.1, it was concluded that formation of a $1: 1$ complex was an appropriate basis for modeling. A test of this postulate was made by fitting the theoretical loading curve (Eq.6-4) to the experimental data, and the results are shown in Fig.7.3. The value of $\beta_{i, d}$ used was obtained by plotting $D$ vs $[N]_{o r g}$ (Fig.7.4). Following Eq. $6-3, \beta_{1,1}=64$ was obtained as the slope of the straight line best fitted to the data points by Iinear regression. It can be seen from Fig.7.4 that the data points do not correspond to a straight line very well. Iinear regression analysis of the data is subject to uncertainty because of this and because the slope of the line is strongly affected by the single point with the highest $D$ value. Nonetheless, the value of $\beta_{1,1}$ obtained this way does seem to fit the experimental data well, as can be seen in Fig.7.3.

The effect of TOMA ${ }^{*}$ on the extraction was investigated as described in section 3.5.5. It was found that the amount of glycerol extracted by TOMA ${ }^{*}$ ions alone is negligible when compared with that extracted by the $\mathrm{NPB}^{-}$extractant. In fact, the amount of extraction is comparable to the partition coefficient of glycerol into 2-ethyl-hexanol, so the observed extraction could be caused by the diluent and not the TOMA*. 
Glycerol

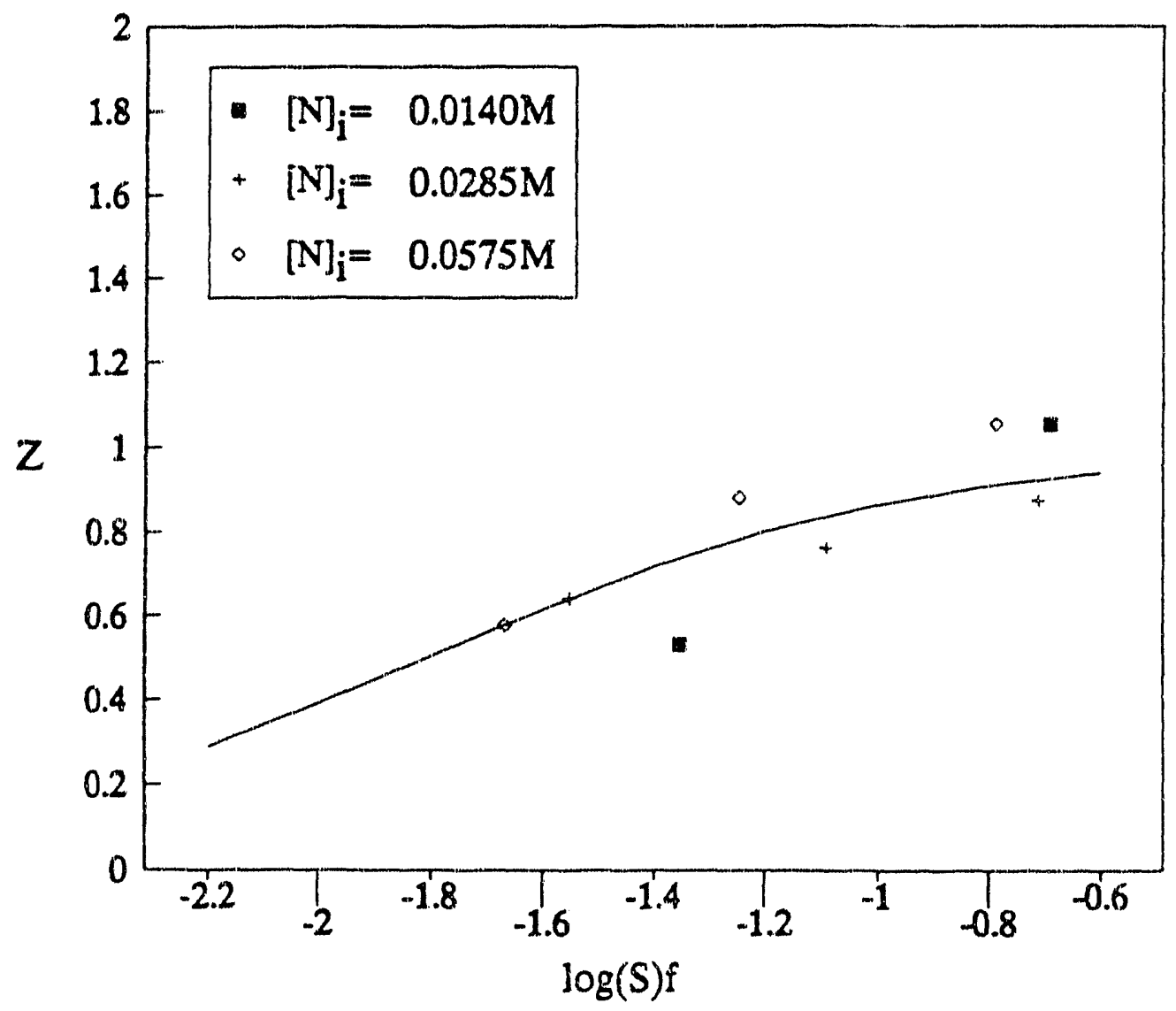

Fig. 7.3 Experimental loading curve for glycerol compared to theoretical loading curve calculated by using Eq. 6-4 
Glycerol

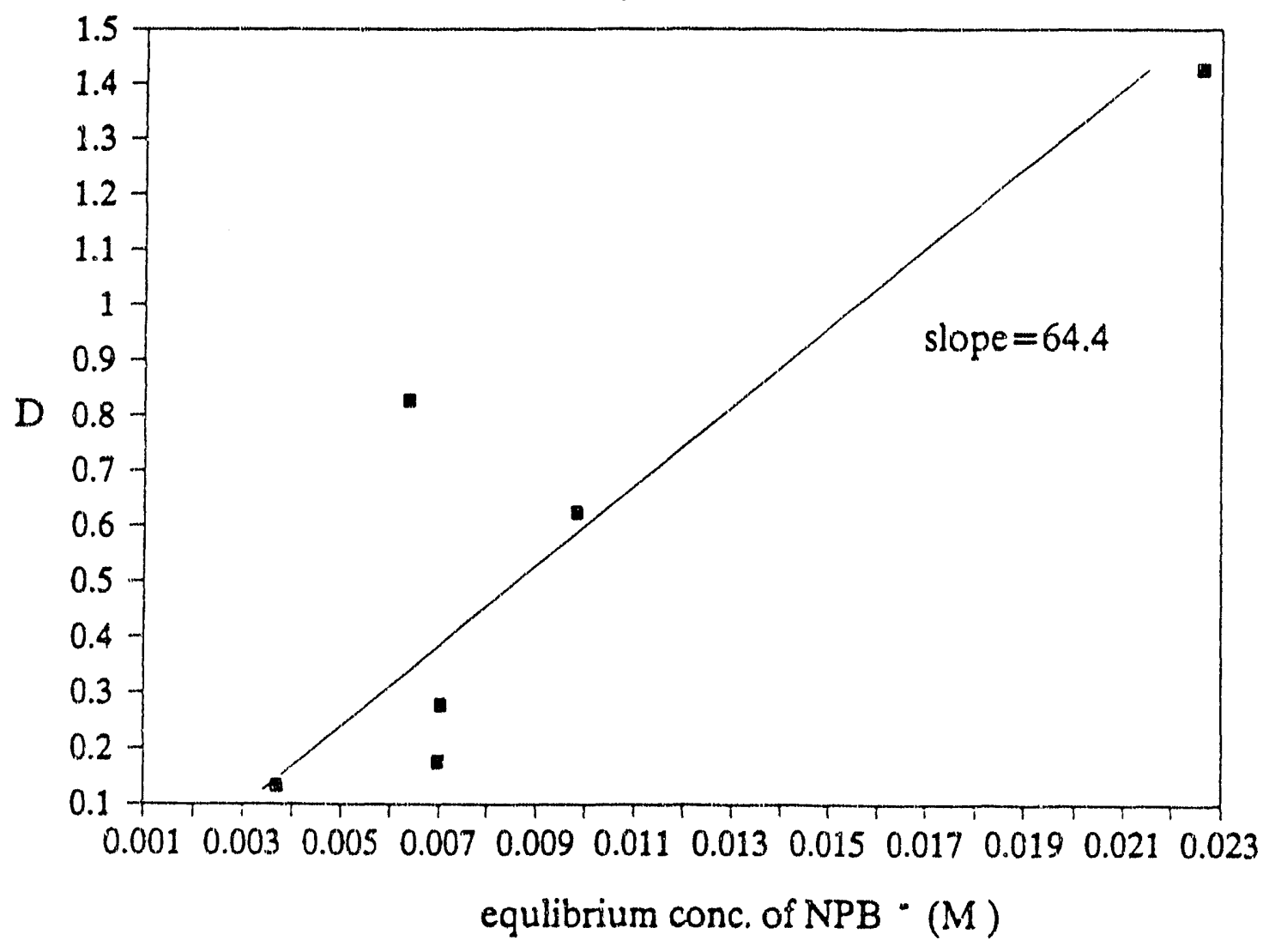

Fig. 7.4 Glycerol extraction results expressed as distribution ratio (D) plotted against equilibrium concentration of NPB in the organic phase 


\subsection{Iiterature data}

Dawber, et al. (1988) derived equilibrium constants from NMR data for complexation of the various solutes used in this work with borate ion in aqueous solution. Table 7.2 lists pertinent values.

TABIE 7.2 Equlibrium constants of complexation $\left(\mathrm{dm}^{2} \mathrm{~mol}^{-2}\right)$ with borate ion, $\mathrm{B}(\mathrm{OH})_{4}^{-}$in aqueous solution (Dawber, et al. 1988)

\begin{tabular}{|c|c|c|}
\hline & $\mathrm{K}_{i}^{\cdot}$ & $\mathrm{K}_{2}{ }^{\cdot}$ \\
\hline 1,2 -propanediol & 4.7 & 0.8 \\
\hline glycerol & 37 & 3.9 \\
\hline fructose & 235 & 111 \\
\hline sorbitol & 540 & 68 \\
\hline
\end{tabular}

* $K_{1}=$ equlibrium constant of $1: 1$ complex (borate:solute) $K_{2}=$ equlibrium constant of $1: 2$ complex ( data obtained by ${ }^{2 i} B$ N.M.R.)

From this information, the complexation constants for 
glycerol and 1,2-propanediol are small compared to those for some other sugars and sugar alcohols. It was felt to be instructive to see if the complexation of these solutes with $\mathrm{NPB}^{-}$anion in organic solutions follows the same trends as the aqueous data. If this is the case, then there could be a correlation between the system we used and the aqueous borate system which has been studied extensively. Therefore we proceeded to do experiments with fructose and sorbitol, which have higher complexation constants with borate in aqueous solution.

\subsection{Fructose and sorbitol}

Fig. 7.5 shows experimentally observed loadings for fructose extraction. Again no dependence of loading upon total NPB ${ }^{-}$concentration and no overloading are observed. A theoretical loading curve for a 1:1 complex was fitted to the experimental data as shown in Fig. 7.6, with the value of $\beta_{1,1}$ $=89.5$ obtained through the distribution ratio graph, Fig.7.7. The shortcomings of the linear regression are similar to those for glycerol. Nonetheless, the theoretical curve agrees quite well with the experimental data from Fig. 7.6. However, a comparison of these data with the glycerol data, Fig. 7.3, show that fructose is not much stronger as a complexant than glycerol, contary to what is predicted from the aqueous borate data. 


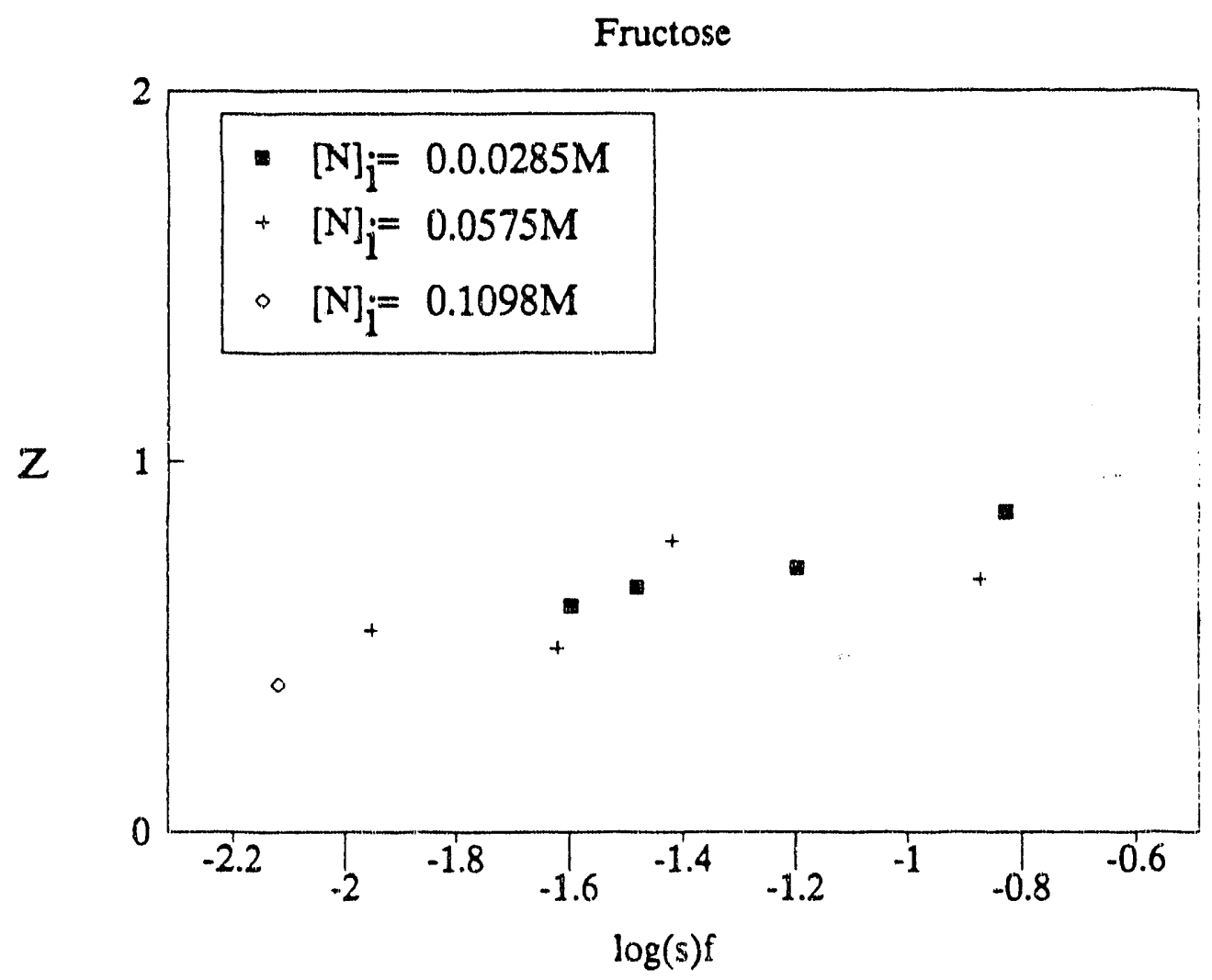

Fig. 7.5 Extraction results for fructose 


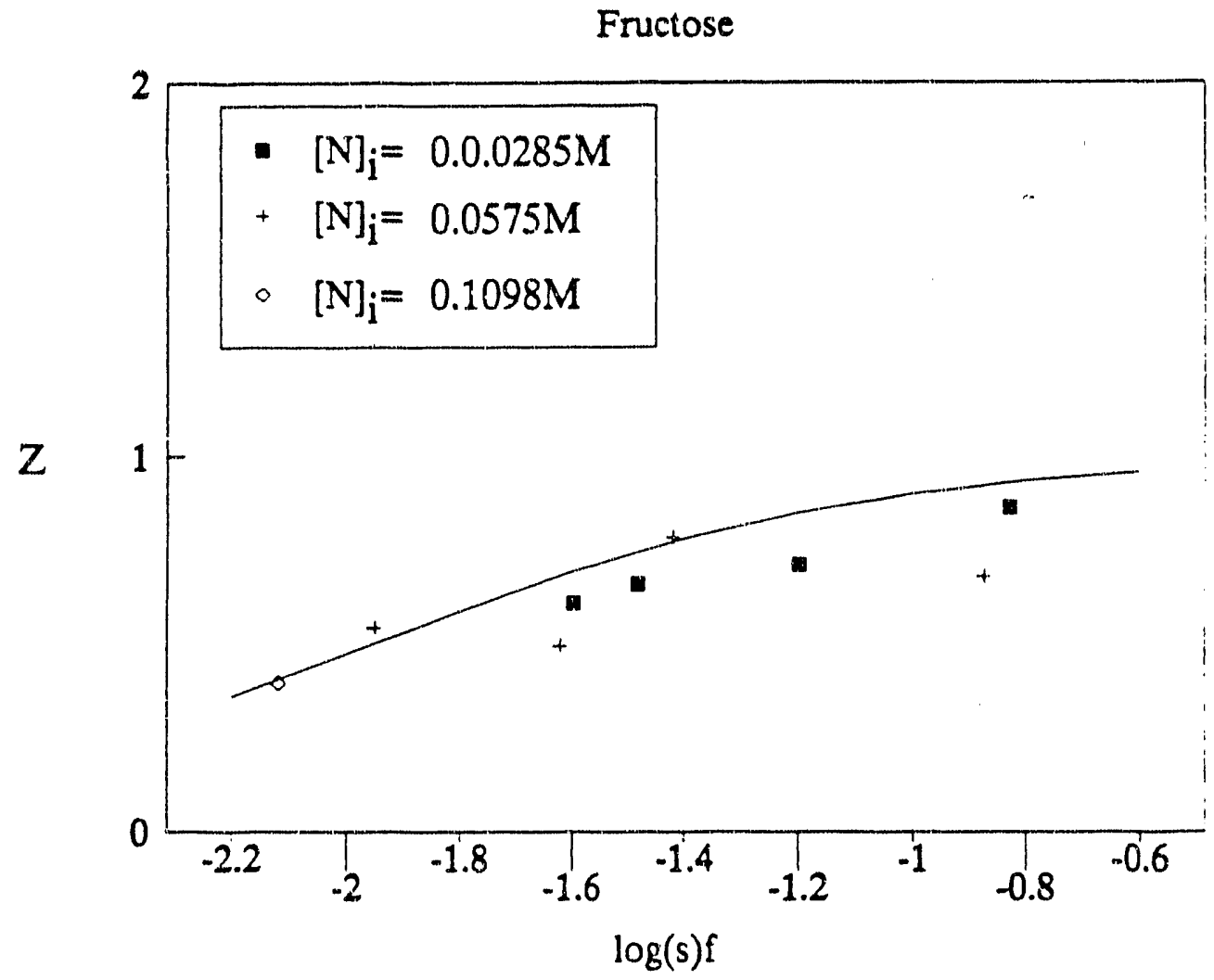

Fig. 7.6 Experimental loading curve for fructose compared to theoretical loading curve calculated by using Eq. 6-4 


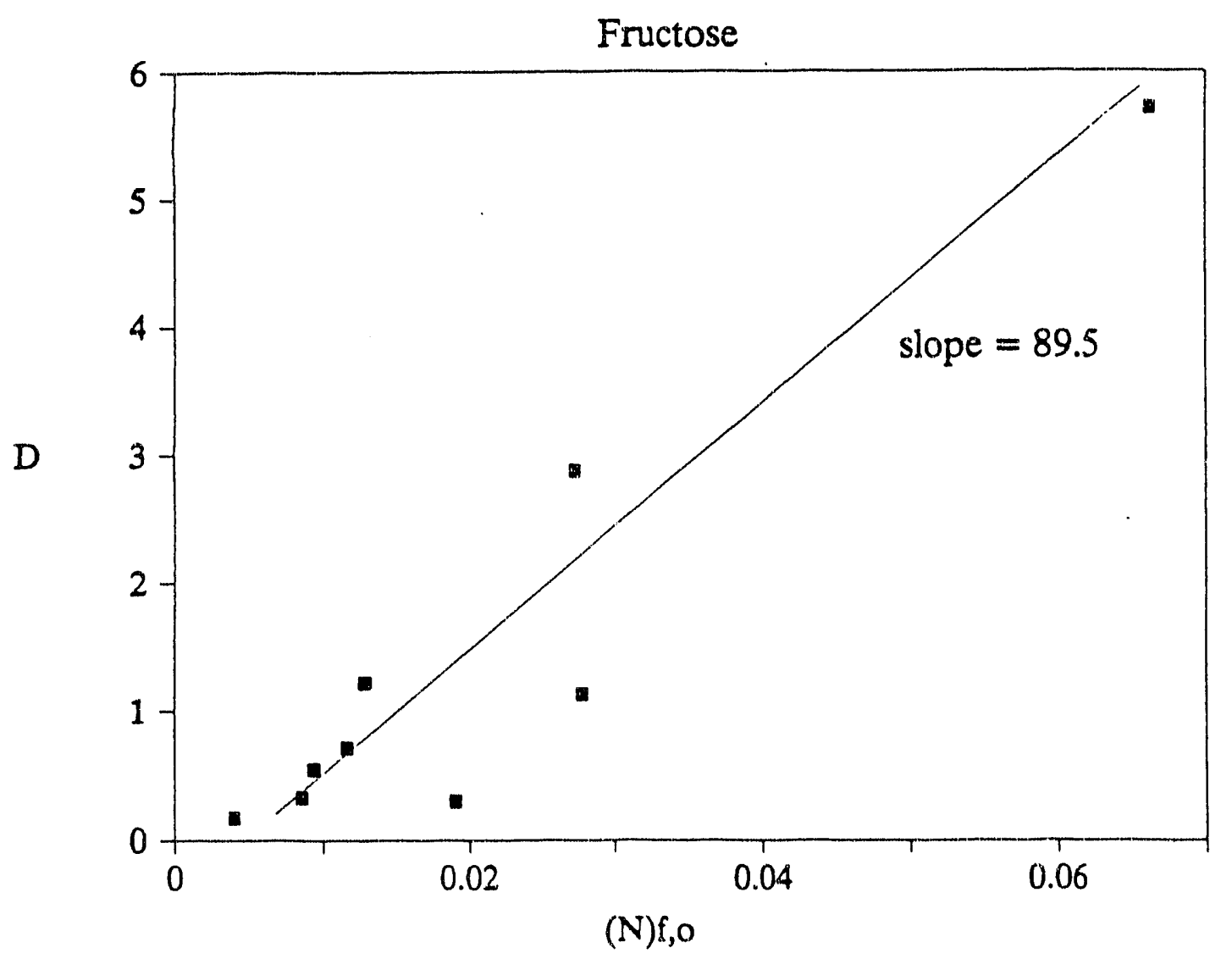

Fig. 7.7 Fructose extraction results expressed as distribution ratio (D) plotted against the equlibrium concentration of NPB in the organic phase 


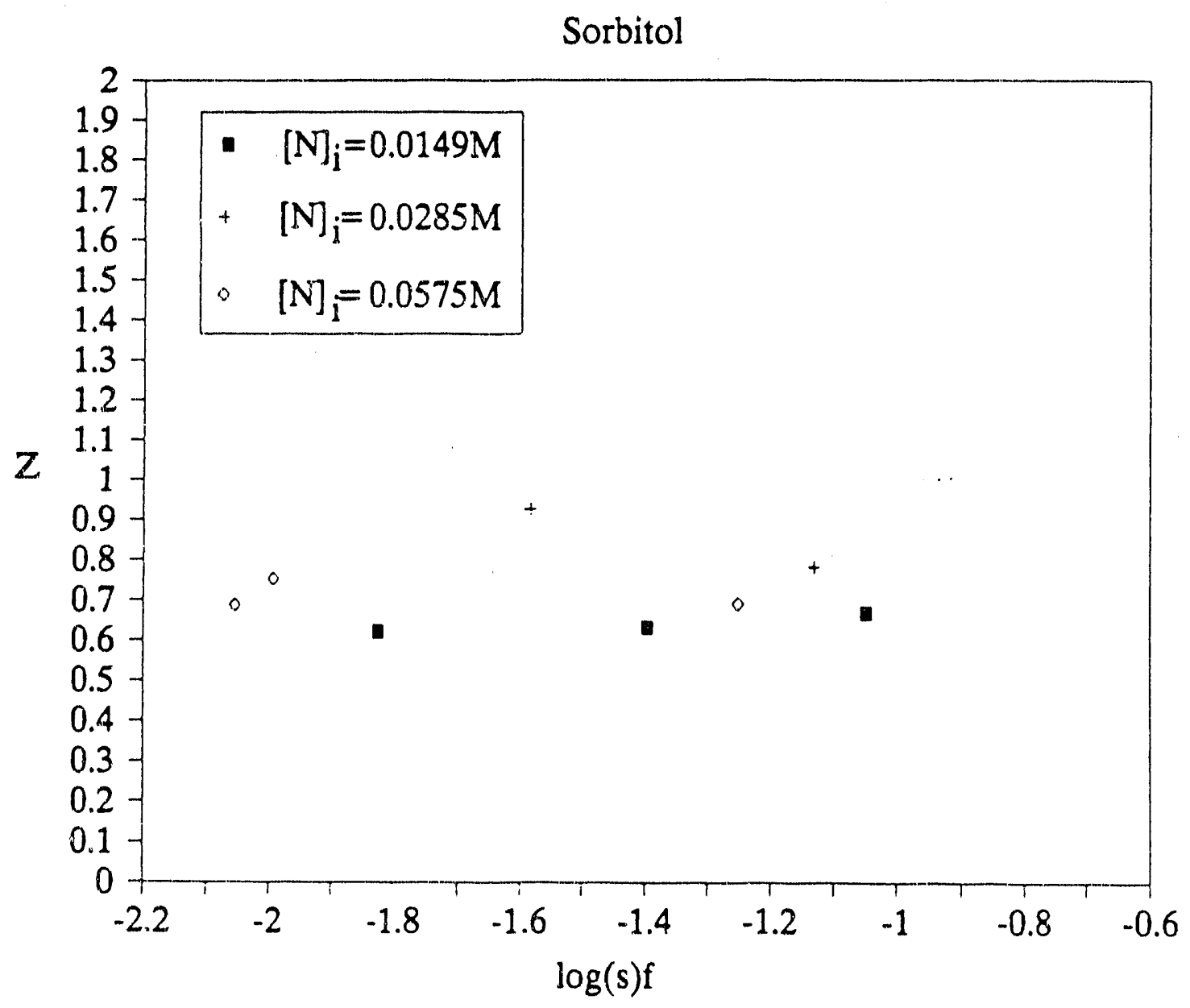

Fig. 7.8 Extraction results for sorbitol expressed in the form of loading curve 
The loading curve for sorbitol is presented in Fig. 7.8, which again suggests that sorbitol may form a $1: 1$ complex with $\mathrm{NPB}^{-}$. The value of $\beta_{1,1}=220$ was obtained from Fig. 7.9 as before and the experimental data are compared with the theoretical loading curve in Fig. 7.10. It can be seen that the theoretical curve does not fit the experimental data as well as is the case for the other solutes. The data are flatter at a value of $z>1$ than the $1: 1$ complex model suggests. By fitting other values for $\beta_{1,:}$ in the theoretical loading curve, it was found that $\beta_{1,1}=162.5$ fits the experimental data better (Fig. 7.11) .

\subsection{1,2-Propanediol}

\subsubsection{Forward extraction}

The experimental results for 1,2 -propanediol extraction are shown in Fig. 7.12 as a loading curve. There are a few observations worth discussing: 1) The degree of loading is much higher than those obtained in previous work under the same conditions (Randel, 1991); 2) there is no dependence of loading upon total $\mathrm{NPB}^{-}$concentration; 3) there is overloading, i.e., more than one mole of 1,2-propanediol is complexed to one mole of NPB-ions. 


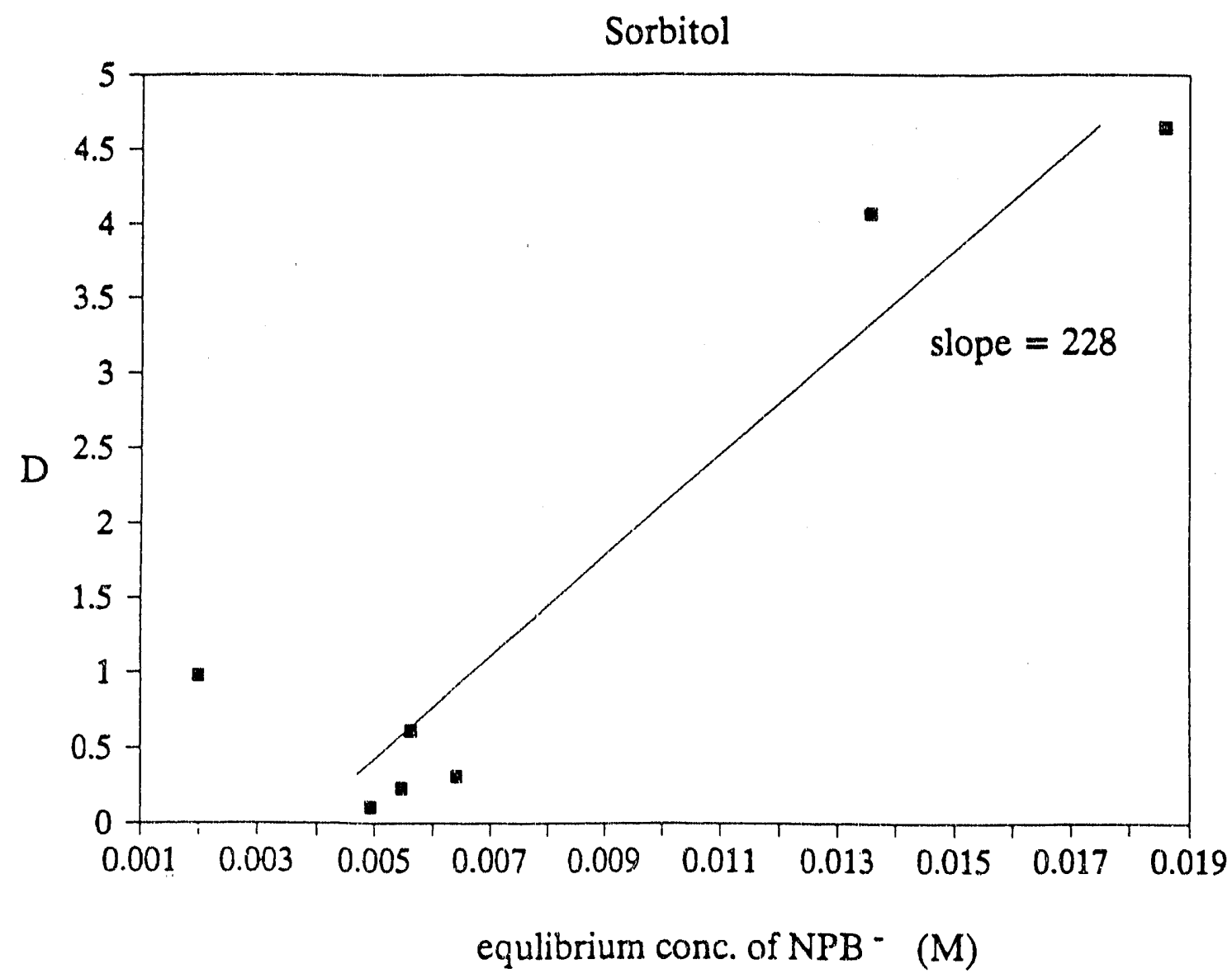

Fig. 7.9 Sorbitol extraction results expressed as distribution ratio (D) plotted against the equlibrium concentration of NPB in the organic phase 
Sorbitol

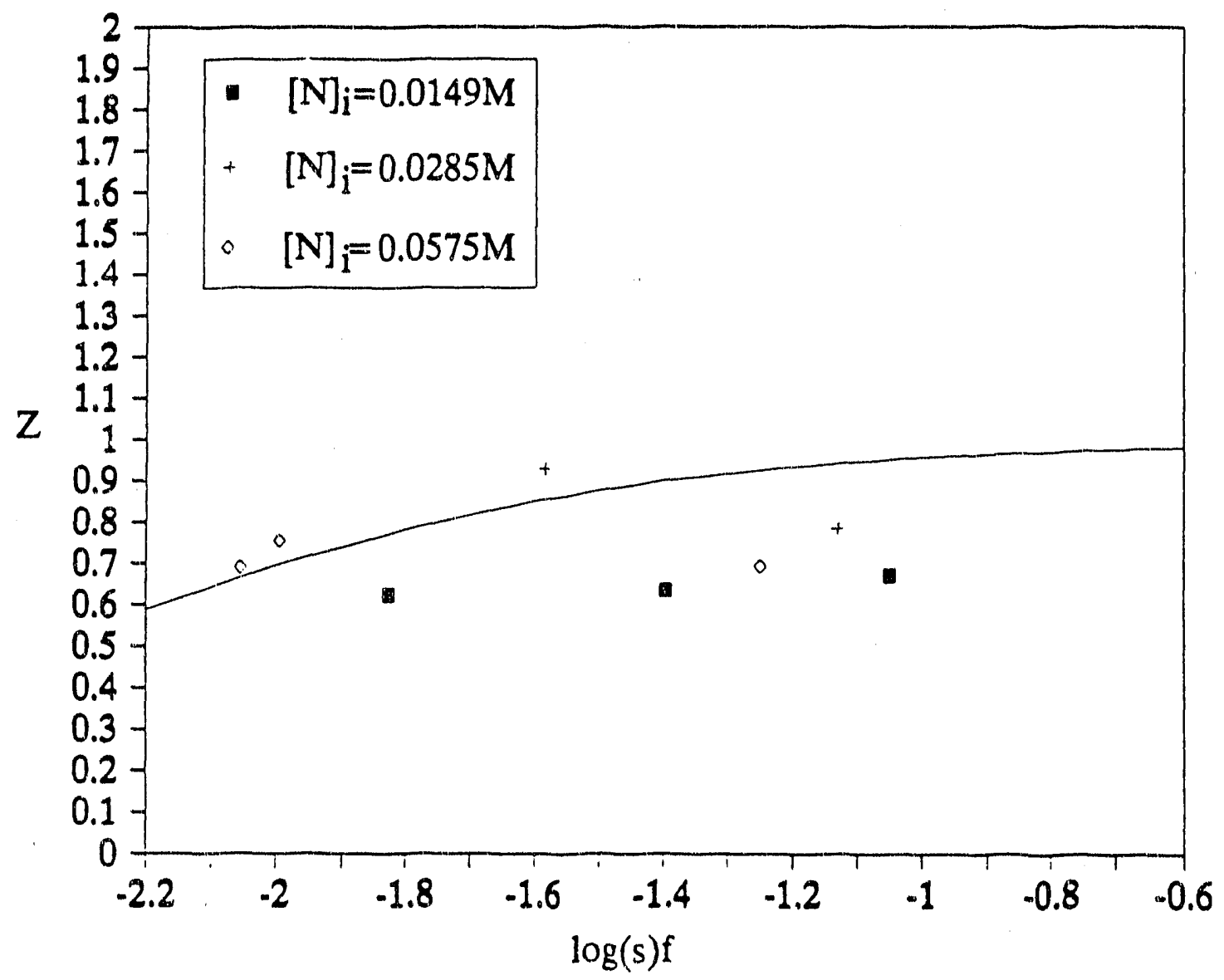

Fig. 7.10 Experimental loading curve for sorbitol compared to theoretical loading curve calculated by using Eq. 6-4 


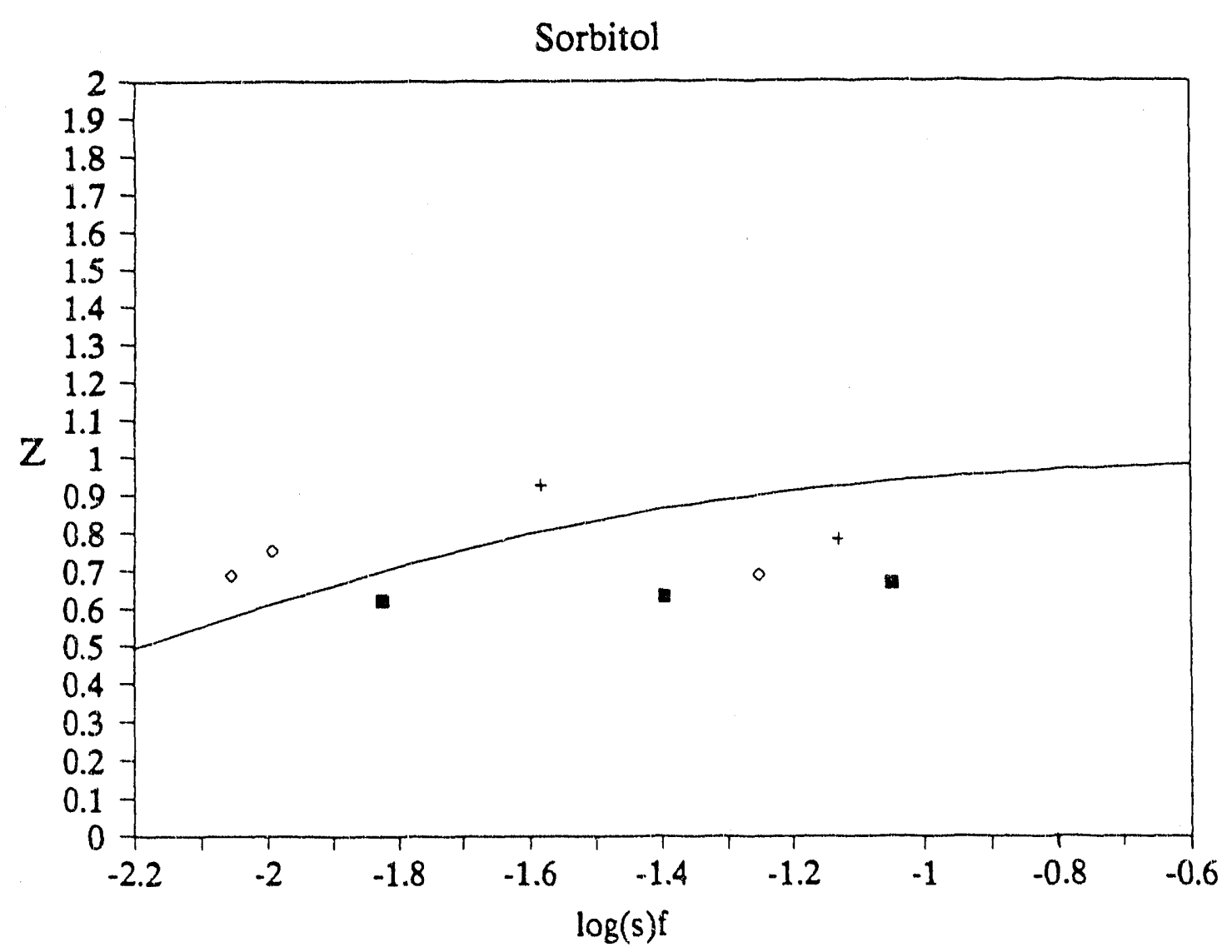

Fig. 7.11 Fitting theoretical loading to experimental data (equlibrium constant $=162.5 \mathrm{dm}^{3} \mathrm{~mol}^{-1}$ ) 


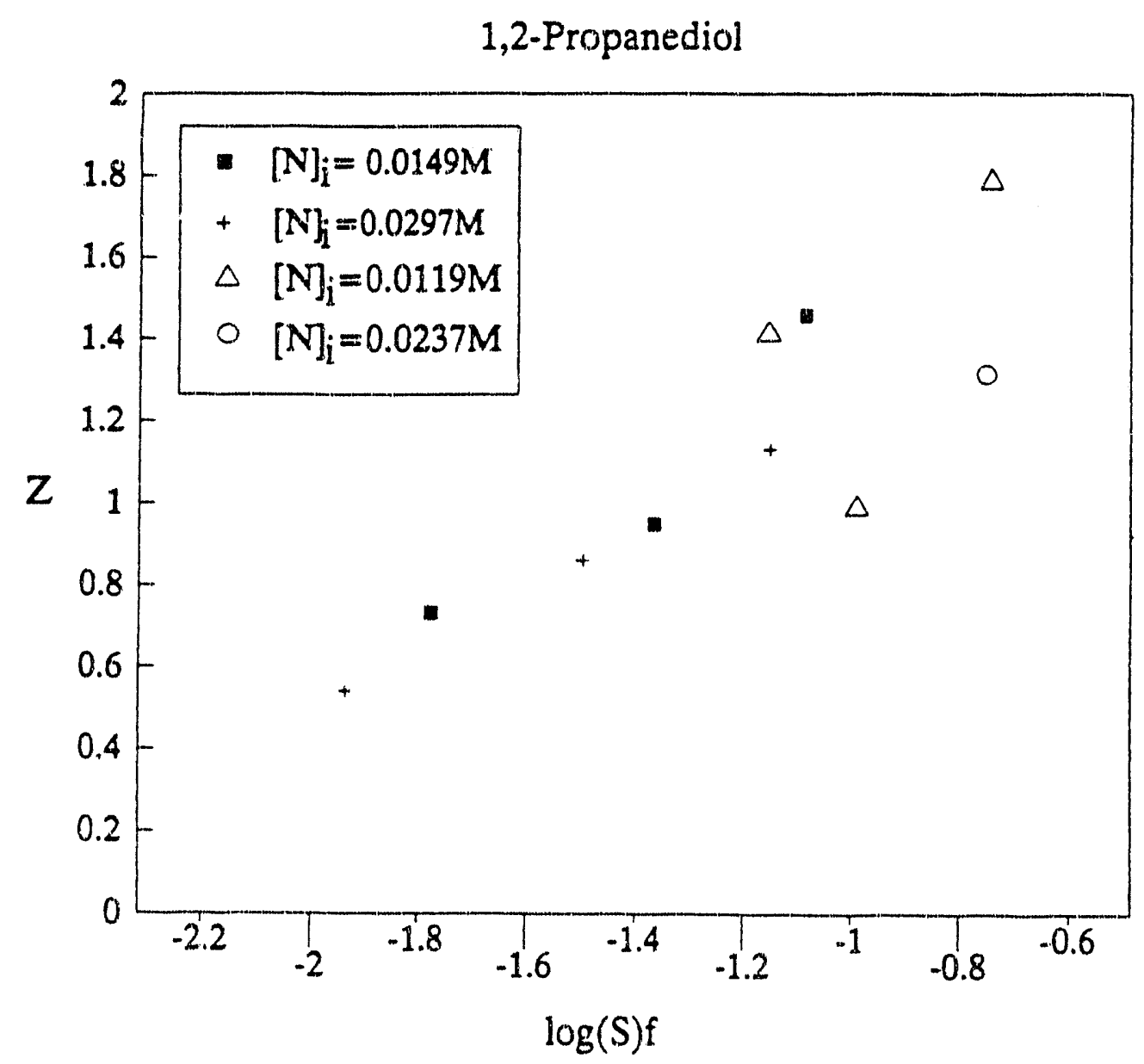

Fig. 7.12 Extraction results for 1,2-propanediol expressed in the form of a loading curve 


\section{Higher loading}

The loadings from the results of Randel (1991) for 1,2propanediol range from 0.15 to 0.25 for $\log (S)$ ag equals -1.0 to -1.5 . This is very much lower than the present data. The higher capacity of the extractant used in this work appears to be due to a more severe initial treatment with NaOH solution (see Chapter 5), resulting in a higher degree of NPBA ionization to $\mathrm{NPB}^{-}$which is believed to be responsible for the complexation. This higher loading after more intense treatment by $\mathrm{NaOH}$ further confirms that it is $\mathrm{NPB}^{-}$, not NPBA, that is effective in the complexation reaction.

No dependence upon $[\mathrm{N}]_{i}$

It can be seen from Fig. 7.12 that the points do not separate into different curves due to different initial NPBconcentrations. For systems with only one $\mathrm{NPB}^{-}$per complex, there should be no effect of total NPB concentration on the loading (Tamada, 1989). Therefore, we believe that the complexes formed between 1,2-propanediol and NPB- anions involve just a single molecule of $\mathrm{NPB}^{-}$per complex.

\section{Overloading}

Loading greater than unity is observed at higher 
equilibrium aqueous concentrations of 1,2-propanediol. This may be due to the use of the third "uncomplexed" -OH group on the $\mathrm{NPB}^{-}$anion after the other two $-\mathrm{OH}$ groups are used in complexation with one molecule of 1,2-propanediol. Selfassociation between the 1,2-propanediol molecules by hydrogen bonding would not be a likely explanation since each 1,2propanediol molecule must use both of its -OH groups to complex with $\mathrm{NPB}^{-}$ion, and there is no excess -OH group for hydrogen bonding. Based on the above explanation, it would also be possible for the other solutes investigated to overload. The fact that they did not show this property may be due to steric hindrance since $\mathrm{TOMA}^{+}$and $\mathrm{NPB}^{-}$are large molecules which form a bulky ion pair, and it would be difficult to load more than one molecule of the solute on the ion pair if the solute is large. Since 1,2-propanediol is the smallest among the solutes investigated, its overloading property supports the postulated explanation.

\subsubsection{Back Extraction}

Back extraction experiments were performed as described in section 3.5.3, where the 1,2-propanediol from loaded organic phase was back-extracted into an aqueous phase consisting of pure water. The results are presented in Fig. 7.1.3 together with the forward extraction results. Back extraction was carried out to check whether the samples had 


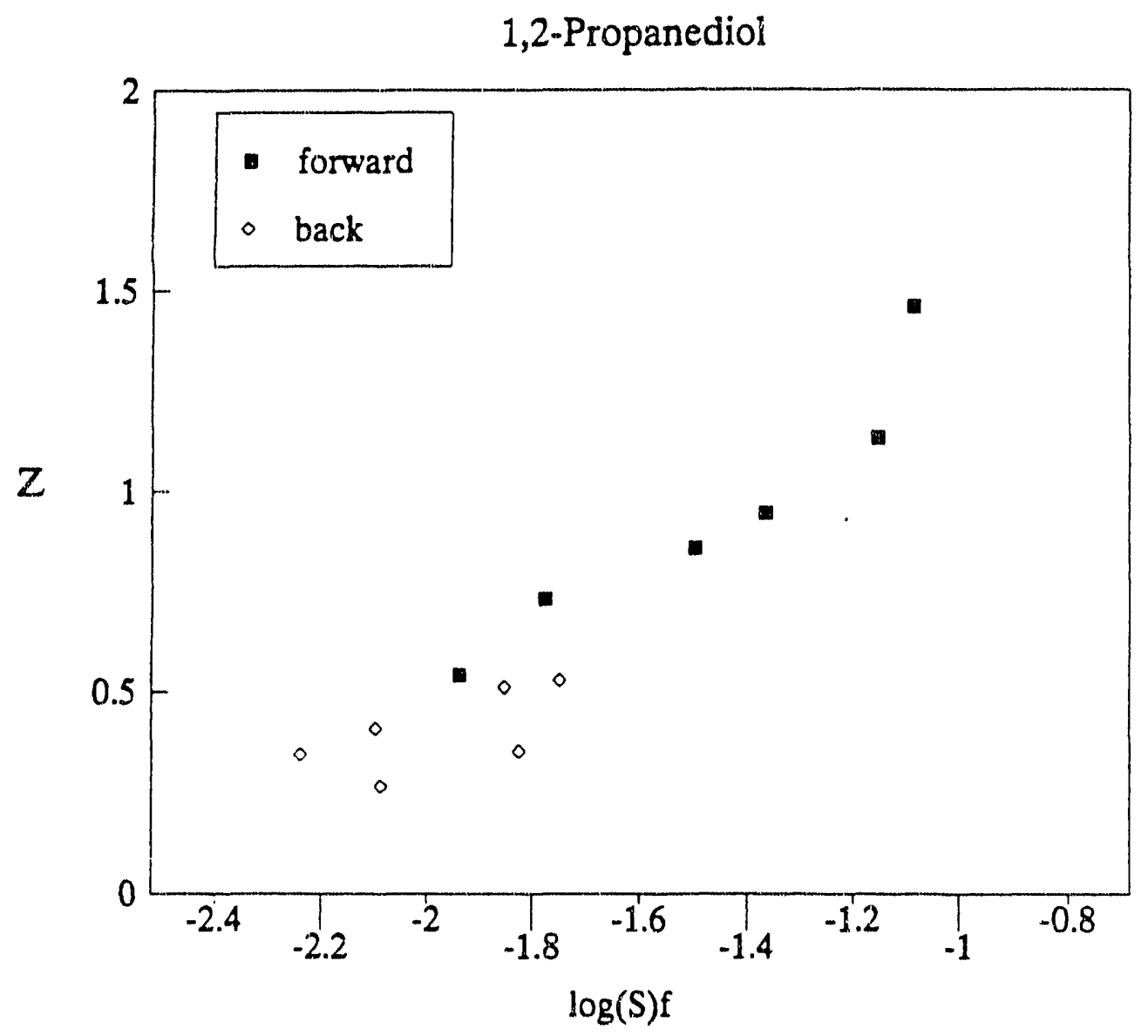

Fig.7.13 Comparing results of forward extraction with back extraction for 1,2-propanediol 
reached equlibrium within the time of contact allowed. If equlibrium had been reached, then the conditions would be the same regardless of from which direction the equlibrium had been approached, and the data points from both the forward and back extraction on the loading graph should lie on the same curve. The fact that the data points from back extraction do lie on or even somewhat below the same curve as those obtained from forward extraction in Fig. 7.13 suggests that the extractions investigated had reached equilibrium, and that the data obtained are therefore useful.

\subsubsection{Effect of diluent}

As described in Section 3.5.4, the effect of 2-ethyl-1hexanol as a diluent on the complexation reaction was tested by changing the diluent to toluene. The extraction experiments were then repeated with all conditions kept the same. It was thought that the 2-ethyl-1-hexanol might compete with the solute for reaction sites on the NPB anion since the diluent itself is an alcohol which possesses $-\mathrm{OH}$ groups. Fig. 7.14 compares the loading curves obtained using the two diluents. It is obvious that the degree of extraction using toluene as the diluent is less than that when 2-ethyl-1-hexanol is used. This shows that the ability of 2-ethyl-1-hexanol to solvate the complex more than overccmes any competition for reaction sites. Therefore, 2-ethyl-1-hexanol appears to be a reasonably 


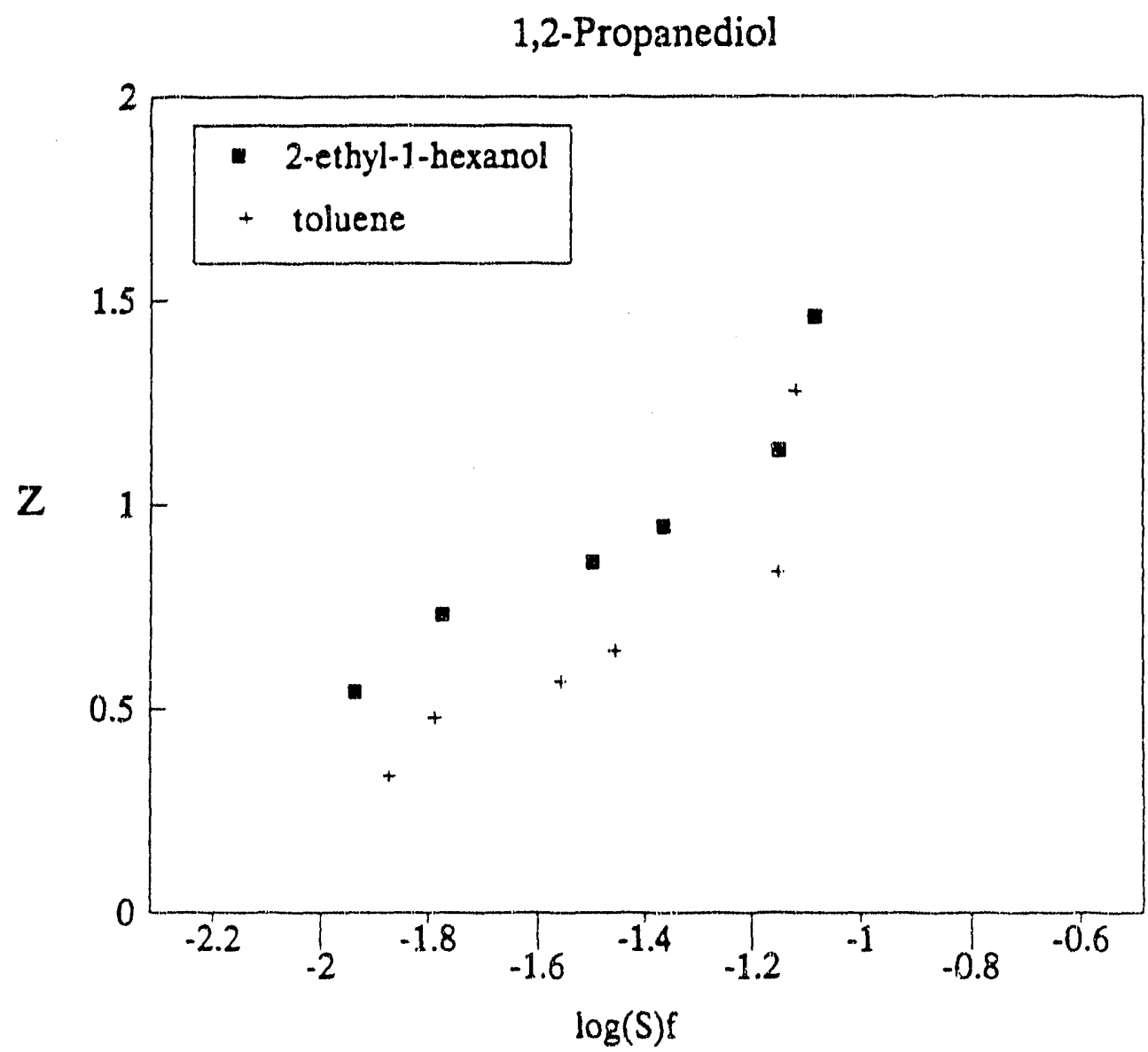

Fig. 7.14 Comparing extraction results for

1,2 propanediol obtained by using two different diluents 
good diluent, and it was used for all other extraction experiments.

\subsection{Lactic acid}

Lactic acid was the final solute investigated. Fig. 7.15 shows the extraction results in the form of a loading curve. It can be seen that very high loading was achieved and that overloading was observed for the entire range of solute concentration investigated. It is known that lactic acid has a very high complexation constant with aqueous borate anion (Friedman, et al., 1974). The necessary-OH groups are thought to be supplied by hydration of $-\mathrm{COOH}$ to $-\mathrm{C}(\mathrm{OH})_{3}$ (Zittle, 1951).

There are several possible explanations for the consistent overloading behavior of lactic acid.

1. It is known that lactic acid self-associates very well due to fairly strong hydrogen bonding between the molecules, which could be one of the causes of overloading. However, after an acid molecule has complexed with the $\mathrm{NPB}^{-}$anion, association of its - $\mathrm{COOH}$ group with another lactic acid molecule may no longer be as facile.

2. Another possible reason would be ester formation between the third uncomplexed $-\mathrm{OH}$ on the NPB- molecule 
Lactic Acid

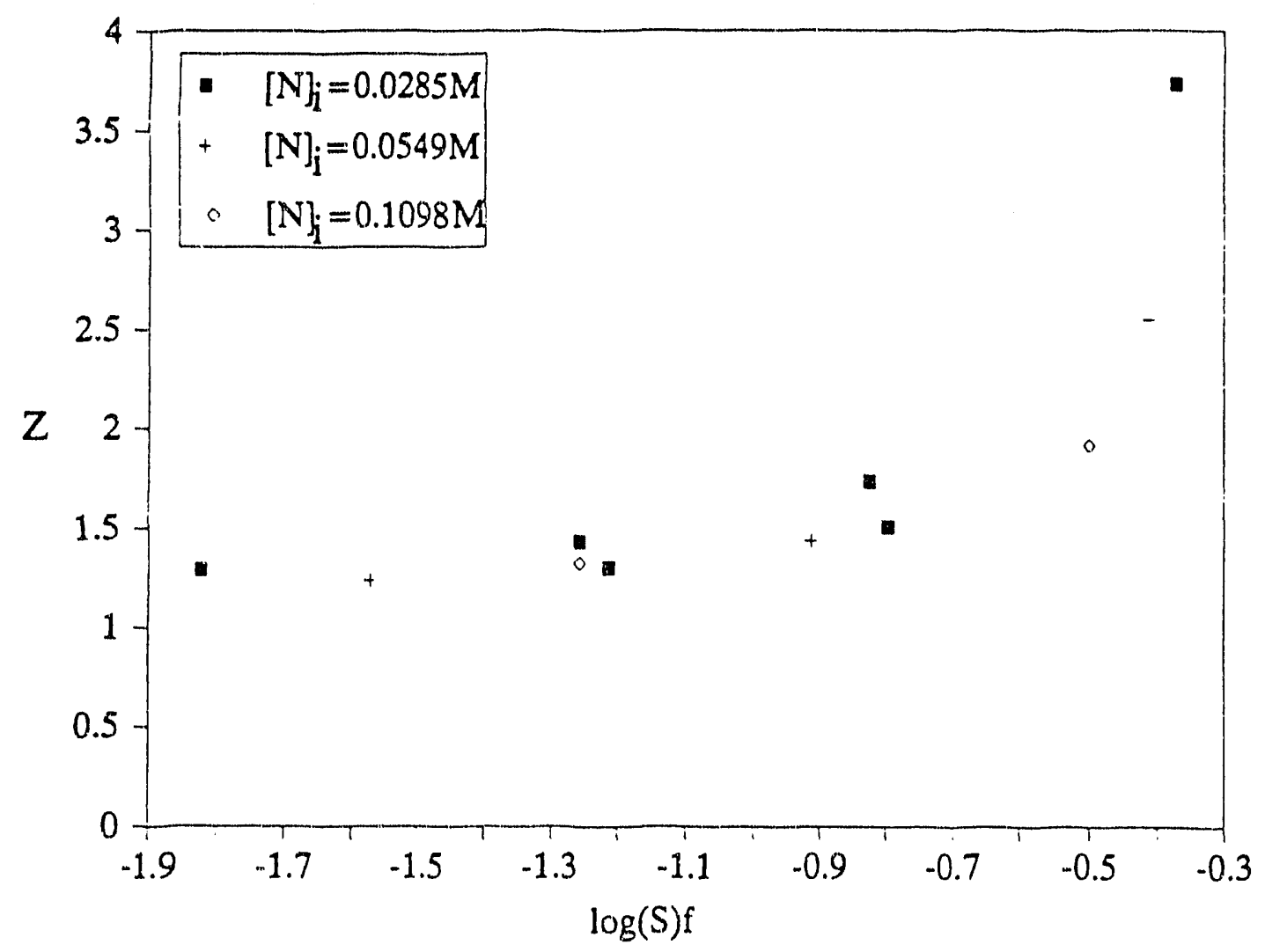

Fig.7.15 Lactic acid extraction results expressed in the form of a loading curve 
and the carboxyl group of the lactic acid molecule. However, it is known that 2-ethyl-1-hexanol stabilizes the 1,1 complex in preference to the 2,1 complex in amine-carcoxylic acid systems (Tamada,1989). This is because 2-ethyl-1-hexanol is capable of donating protons, which compete with the carhoxyl protons of the second acid for the carboxylate binding site on the first acid complexed to the NPB'. This competition tends to destabilize the 2,1 complex.

3. The most probable reason for overloading would be a high degree of extraction by something other than NPB' present in the extractant. This leads to the concept that the excess TOMA ${ }^{+}$ions left unpaired with $\mathrm{NPB}^{-}$ anions in the extractant are also participating in the extraction, since quaternary ammonium compounds are known to be highly effective in strong base anionic exchange resins for separation of organic acids.

Experiments as described in Section 3.5.5 were done; and the results obtained proved complexation of lactic acid with TOMA ${ }^{+}$. Table 7.3 shows some sample results for lactic acid extraction by TOMA ${ }^{+}$ions. These results show that, under the same extraction condition, TOMA ${ }^{*} \mathrm{Cl}^{-}$contributes 30-508 of the extraction by the TOMA ${ }^{+} N B^{-}$ion-pair extractant. The lactic acid molecules can give up the $\mathrm{H}^{+}$ions readily to form lactate 
anion, which then pairs with the TOMA ${ }^{+}$i.e., a strong acidbase interaction.

TABIE 7.3 Comparison of results for lactic acid extraction by NPB- extractant and $\operatorname{TOMA}^{+} \mathrm{Cl}^{-}$Only

\begin{tabular}{|l|l|l|l|l|}
\hline & initial & {$[S]=0.2 \mathrm{M}$} & initial & {$[\mathrm{S}]=0.1 \mathrm{M}$} \\
\hline & $\begin{array}{l}{[\mathrm{S}] \mathrm{i}-} \\
{[\mathrm{S}] \mathrm{f}}\end{array}$ & $\begin{array}{l}\delta \\
\text { extrac. }\end{array}$ & $\begin{array}{l}\text { [S]i- } \\
{[S] f}\end{array}$ & $\begin{array}{l}8 \\
\text { extrac. }\end{array}$ \\
\hline \hline NPB" extractant & 0.0514 & 25.53 & 0.0387 & 38.73 \\
\hline $\left.\mathrm{TOMA}^{+} \mathrm{Cl}\right]^{-}$only & 0.0148 & 14.80 & 0.0260 & 12.90 \\
\hline
\end{tabular}

For extraction by $\operatorname{TOMA}^{-1} \mathrm{Cl}^{-}$alone, it was found that at lower solute concentrations, approximately $1: 1$ solute to TOMA ${ }^{+}$ ratio was obtained and at higher solute concentrations, overloading was observed. Loading of more than one mole of lactic acid per mole of $\mathrm{TOMA}^{+} \mathrm{Cl}^{-}$present was probably due to self-association between the lactic acid molecules. However, it is hard to separate the lactic acid extraction by TOMA $^{+}$ from the extraction by NPB" for modeling purposes, because of unreliable assumptions. Since the $\mathrm{pK}_{\mathrm{a}}$ of lactic acid is 3.85 compared to 7.1 for NPBA, the TOMA ions in the organic 
extractant should preferentially bind to lactic acid rather than NPB". Therefore, we can presume that at low solute concentrations lactic acid binds to the $\mathrm{TOMA}^{+}$ions in a $1: 1$ ratio. Using this assumption, the loadings of lactic acid extracted by $\mathrm{NPB}^{-}$alone were calculated. The new loading curve is shown in Fig. 7.16. The new loading curve retains the shape of the loading curve in Fig. 7.15 but the plateau has shifted to a value of loading around 1 .

\subsection{Discussion}

The theoretical complexation model fits the experimental results reasonably well, and the order of complexation constants for the solutes follows trends similar to those found for complexation with borate anions in aqueous conditions (Table 7.4). The results also showed that by incorporating $\mathrm{NPB}^{-}$into the diluent, extraction of the solutes is significantly enhanced. Distribution ratios as high as 5.7 were achieved (fructose).

For all of the solutes investigated, there appears to be only one $\mathrm{NPB}^{-}$per complex. For glycerol, fructose and sorbitol, the ratio of solute molecules to $\mathrm{NPB}^{-}$in the complexes appears to be 1:1. For 1,2-propanediol, more than one molecule of solute binding to one NPB was observed. This could be due to the use of the free $-\mathrm{OH}$ on the NPB- The other solutes did not overload this way, a fact which may be due to 


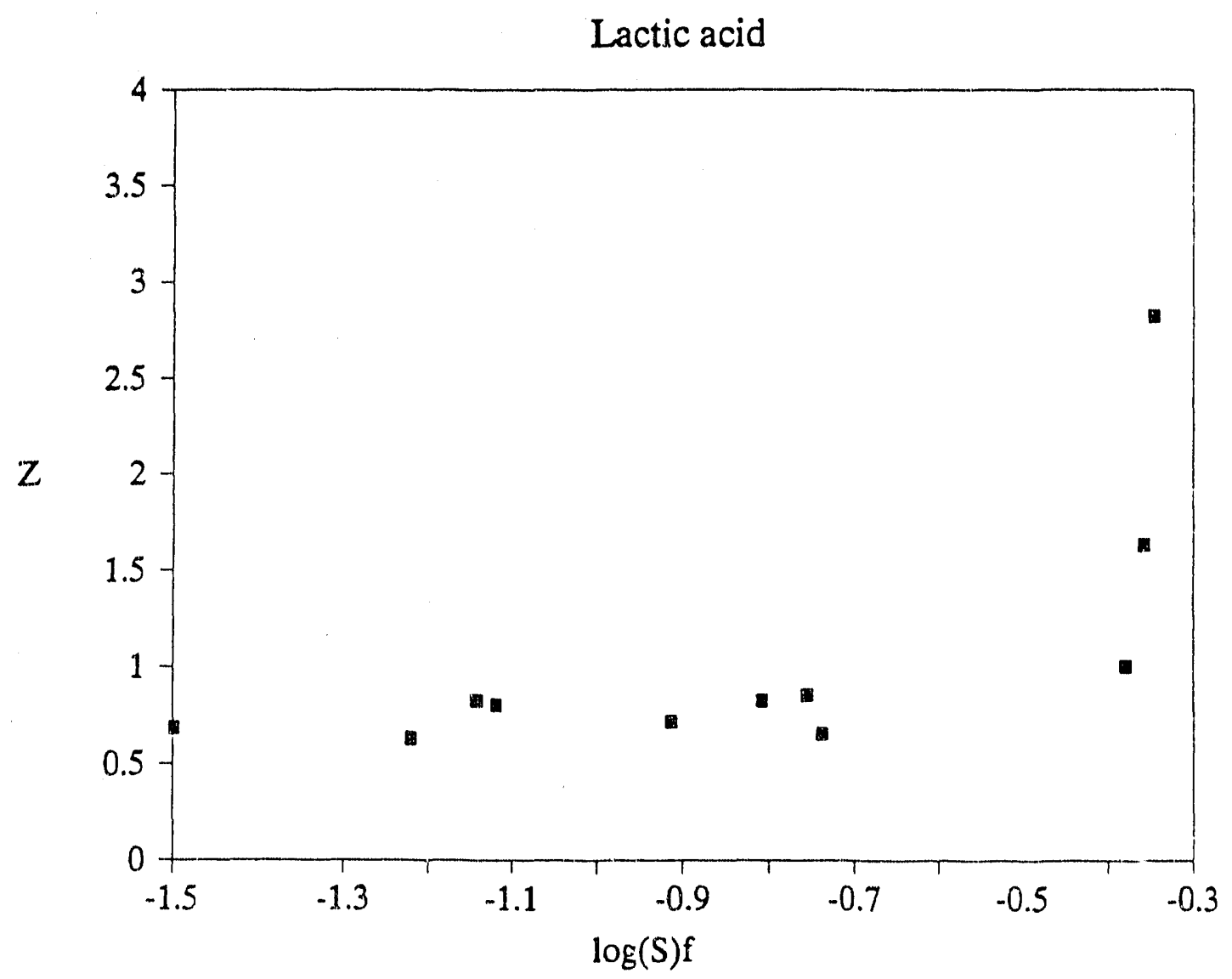

Fig. 7.16 Lactic acid extraction results after effect of TOMA has been removed 
steric hindrance since $\mathrm{TOMA}^{+}$and $\mathrm{NPB}^{-}$are large inolecules which form a bulky ion pair. It would be difficult to load more than one molecule of the solute on the ion pair if the solute is large. 1,2-Propanediol is the smallest among the solutes.

TABIE 7.4 Equlibrium constants of complexation $\left(\mathrm{dm}^{3} \mathrm{~mol} \mathrm{l}^{-1}\right)$ of solutes with borate in aqueous conditions (Dawber, et al, 1988 ) and with NPB - in organic conditions

\begin{tabular}{|l|l|l|}
\hline & $\mathrm{B}(\mathrm{OH})_{4}^{\circ}$, aqueous & $\mathrm{NPB}^{-}$, organic \\
\hline glycerol & 37 & 64.3 \\
\hline fructose & 235 & 89.5 \\
\hline sorbitol & 540 & 162.5 \\
\hline
\end{tabular}

\subsection{Recovery}

It is known that for complexes with borate ions under aqueous conditions, the reaction can be reversed very easily (Zittle, 1951). A preliminary experiment was done whereby a sorbitol-loaded organic extractant was contacted with equal volumes of $0.01 \mathrm{~N}$ sulfuric acid, and the results showed $50 \%$ to 908 recovery (Table 7.5 ). 
TABIE 7.5 Results for sorbitol recovery experiments

\begin{tabular}{|c|c|c|}
\hline$[S]_{i}$ & (S)..2 & \& recovered \\
\hline 0.050 & 0.025 & 49.7 \\
\hline 0.041 & 0.022 & 53.1 \\
\hline 0.026 & 0.024 & 94.0 \\
\hline
\end{tabular}

$[S]_{i, 0}=$ initial sorbitol concentration in the organic phase

$(S)_{t, a q}=$ equlibrium sorbitol concentration in the aqueous phase

\subsection{Implementation}

This is part of an early stage of investigation of the process feasibility using NPBA for extraction of $-O H$ bearing solutes from dilute aqueous solutions. More data are required before making any important conclusions regarding process implementation. However, the data obtained in this work showed a substantial loss of NPBA to the aqueous phase during initial treatment with alkali solutions, which would not be economical in an industrial process. One approach for overcoming this problem could be to incorporate the NPBA onto a solid support, such as an adsorbent. 


\section{REFERENCES}

Areson, D.R. 1989. Separation of low molecular weight alcohols fron dilute aqueous solutions by reversible chemical complexation. Ph.D. Dissertation, Department of Chemical Engineering, Unversity of California, Berkeley.

Babcock, $I$. and R. Pizer. 1980. Dynamics of boron acid complexation reactions. Formation of $1: 1$ boron acid-ligand complexes. Inorg.Chem. 19, 56-61.

Barker, P.E. \& Thawait, S. 1983. Separation of fructose from carbohydrate mixtures by semi-contiuous chromatography. Chem. \& Ind. 21, 817-21.

Boeseken, J. 1949. The use of boric acid for the determination of the configuration of carbohydrates. Adv. Carbohydr. Chem. $4,28-210$.

Busche, R.M. 1987. Recovering chemical products from dilute fermentation broths. Biotech. Bioeng. Symp. No.13. 597-615.

Cameron, D.C. and C.I. Cooney. 1986. A novel fermentation: The production of $R(-)-1,2$-propanediol and acetol by clostridium thermosaccharolyticum. Biotechnology. 4: 651-654.

Chemical Marketing Reporter. February 9, 1987.

Dawber, J.G. et al. 1988. A polarimetric and ${ }^{21} \mathrm{~B}$ and ${ }^{13} \mathrm{C}$ nuclear magnetic resonance study of the reaction of the tetrahydroxyborate ion with polyols and carbohydrates. $J$. Chem. Soc., Faraday Irans, 1, 1988, 81(1), 41-56.

Dyrssen, D. et.al. 1969. A study of the extraction of boric acid with 2,2-diethylpropanediol-1,3 and 2-ethylhexanediol-1,3 in chloroform. Anal. Chim. Acta. 46, 55-61.

Flick, E.W. Ed. 1985. Industrial Solvents Handbook, 3rd ed. Park Ridge, NJ: Noyes Data Corp.

Friedman, S. et.al. 1974. Complexation of phenylboronic acid with lactic acid: stability constant and reaction kiretics. J. Amer. Chem. Soc., 96, 5381-5384.

Jackson, W.M. and J.S. Drury. 1959. Miscibility of organic solvent pairs. Industrial and Engineering Chemistry, vol 51, no. 12 .

Jaln, M.K., Datta, R. and J.G. Zeikus. 1989. Kigh value organi. acids fermentation-emerging processes and products. 
Bioprovess Engineering: The First Generation. Chap.25. T.K.Ghose, ed.

Karhadkar, P.P. et al. 1990. Pilot scale distillery spentwash biomethanation. J. Env. Eng., 116, 1029-45.

Katsutoshi I., et al. 1984. Solvent extraction of phenol with primary and tertiary amine and a quaternary ammonium compound. Sol. Extr. IOß Exch., 2(7\&8), 1047-1067.

Katsutoshi I., et al. 1986. Solvent extraction of phenol with mineral acid salts of high-molecule-weight amines. Sol. Extr. Ion Exch., 4(2), 199-216.

Kertes, A.S., and C.J.King. 1987. Extraction chemistry of low molecular weight aliphatic alcohols. Chem. Rev. 87, 687-710.

Kim, S.S., et al. 1985. Separation of fructose and glucose by reverse osmosis. Ind. Eng. Chem. Fundam. 1985, 24, 409-412.

King, C.J. 1987. Separation processes based on reversible chemical complexation. Handbook of Separation process Technology. Chap. 15. R.W. Rousseau, ed. NY: Wiley.

Leo, A., C. Hansch, and D. Elkins. 1971. Partition coefficients and their uses. Chemical Reviews. 71(6), 525-616.

Pizer, R. and I. Babcock. 1977. Mechanism of the complexation od boron acids with catechol and substituted catechols. Inorg. Chem. 16, 1677-1681.

Randel, I. A. 1991. Separation of glycols from dilute aqueous solutions via complexation with boronic acids. Masters Thesis, Department of Chemical Engineering, University of California, Berkeley.

Reiche, C.R. and J.A. Heckman. July 20, 1976. Method of producing glycols. U.S.patent No. 3,970,711.

Shinbo, T. et al. 1986. Uphill transport of monosaccharides across an organic liquid membrane. J. Chem. Soc., Chem. Commun. No. $4,349-351$.

Short, J.F. and P. Eaglesfield. 1952. Trans. Inst. Chem. Eng., 30,109 .

Stowell, J.D. et al. Bioactive microbial products, 3: Downstream processing. Arademis Press, Iondon (1986).

Tamada, J., A.S. Kertes, and C.J. King. 1990. Extraction of 
carboxylic acids with amine extractants: 1. Equilibria and law of mass action modelling. Ind. Eng. Chem.. Res., 29, 13191326 .

Tamada, J. and C.J. King. 1990a. Extraction of carboxylic acids with amine extractants: 2. Chemical interactions and interpretations. Ind. Eng. Chem.. Res., 29, 1327-1333.

Tamada, J. and C.J. King. 1990b. Extraction of carboxylic acids with amine extractants: 3. Temperature, water coextraction, and process considerations. Ind. Eng. Chem.. Res., 29, 1333-1338.

Tamada, J.A. 1989. Extraction of carboxylic acids by amine extractants. Ph.D. Dissertation, Department of Chemical Engineering, University of California, Berkeley.

Ward, O.P. 1989. Industrial Chemicals. Fermentation Biotechnology. Chap.8. Open University Press.

Weast, R.C. et al, Ed. 1990. CRC Handbook of Chemistry and Physics, 70th edition, CRC Press Inc.

Zitttle, C.A. 1951. Reaction of borate with substances of biological interest. Advances in Enzymology and Related subjects of Biochenistry, vol XII. F.F.Nord, ed. NY: Interscience. 493-527. 


\section{APPENDIX. TABULATION OF EXPERIMENTAI RESULTS}

Results of the batch extraction experiments for each solute are tabulated separately below in Tables $A-1$ to $A-6$.

\section{List of symbols:}

$(S)_{f, a q}=$ equilibrium concentration of the solute in the aqueous phase

$(S)_{i, a q}=$ initial concentration of the solute in the aqueous phase
$(\mathrm{N})_{i, 0}=$ initial concentration of $\mathrm{NPB}^{-}$ions in the organic extractant

$(\mathrm{N}) /(\mathrm{S})=$ initial ratio of $\mathrm{NPB}^{-}$to solute

$\%$ extrac. = Amount of solute extracted in terms of percentage

$(N)_{4,0}=$ equilibrium concentration of free NPB- in the organic extractant

$D=$ distribution ratio (see Section 5.4)

$z=$ loading (see Section 5.7 ) 


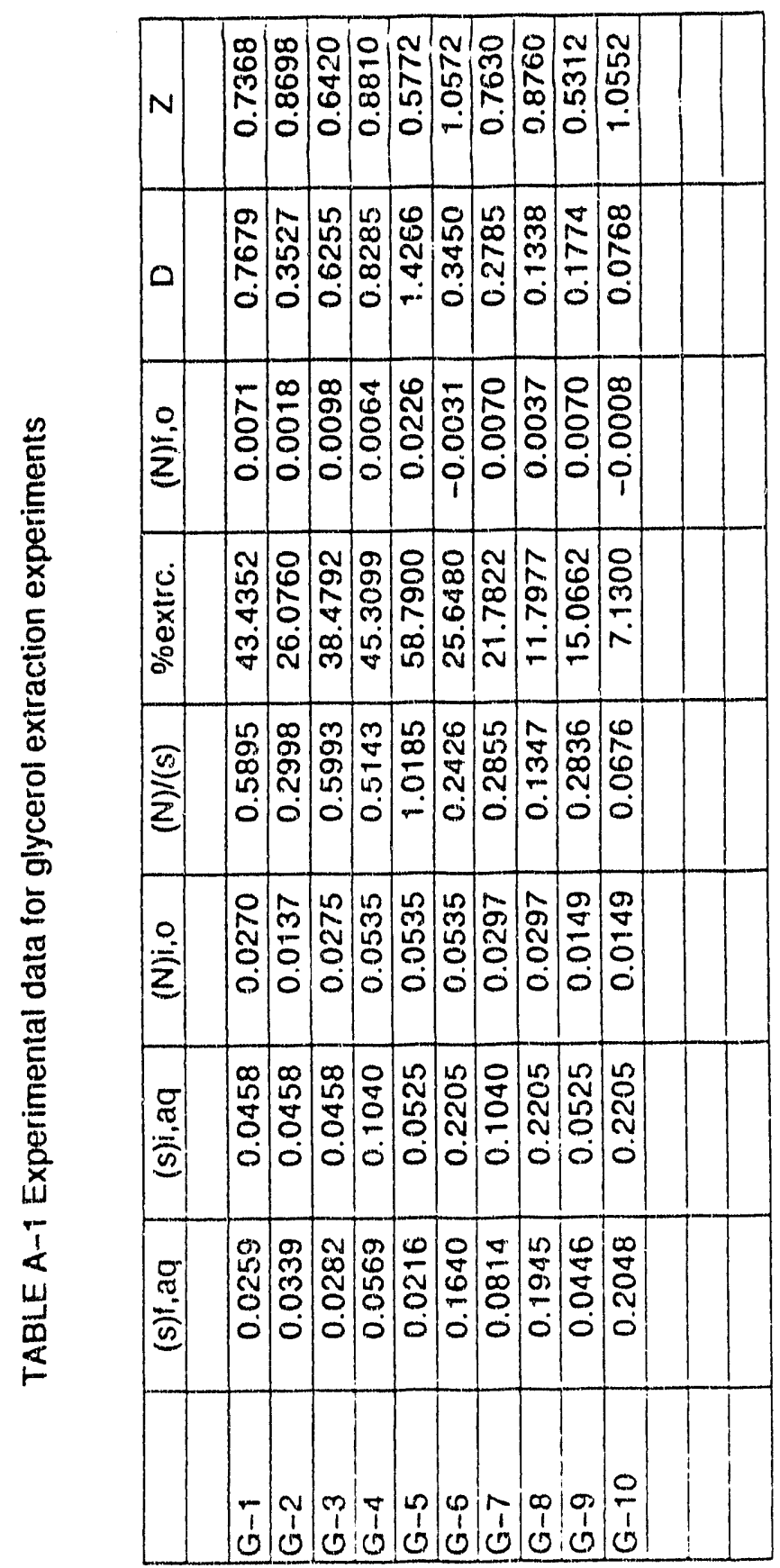




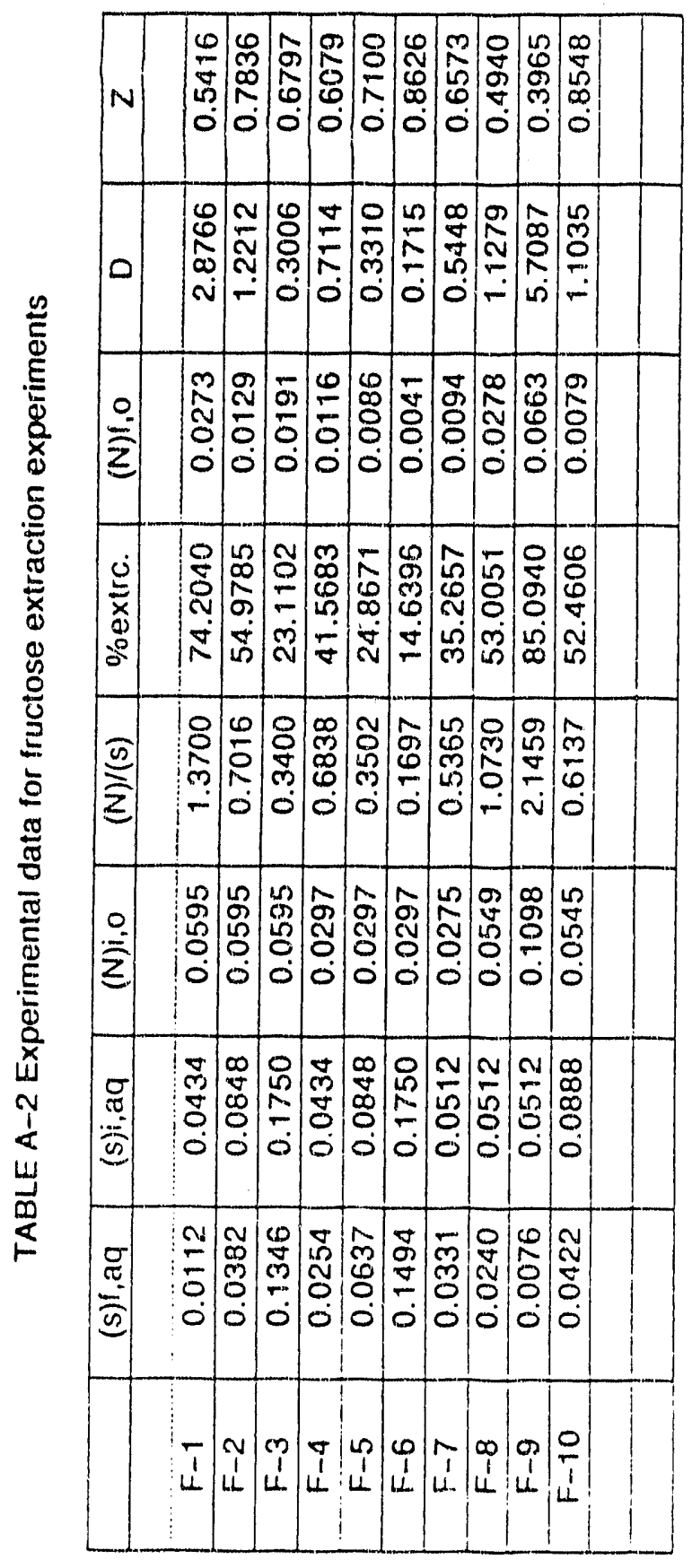




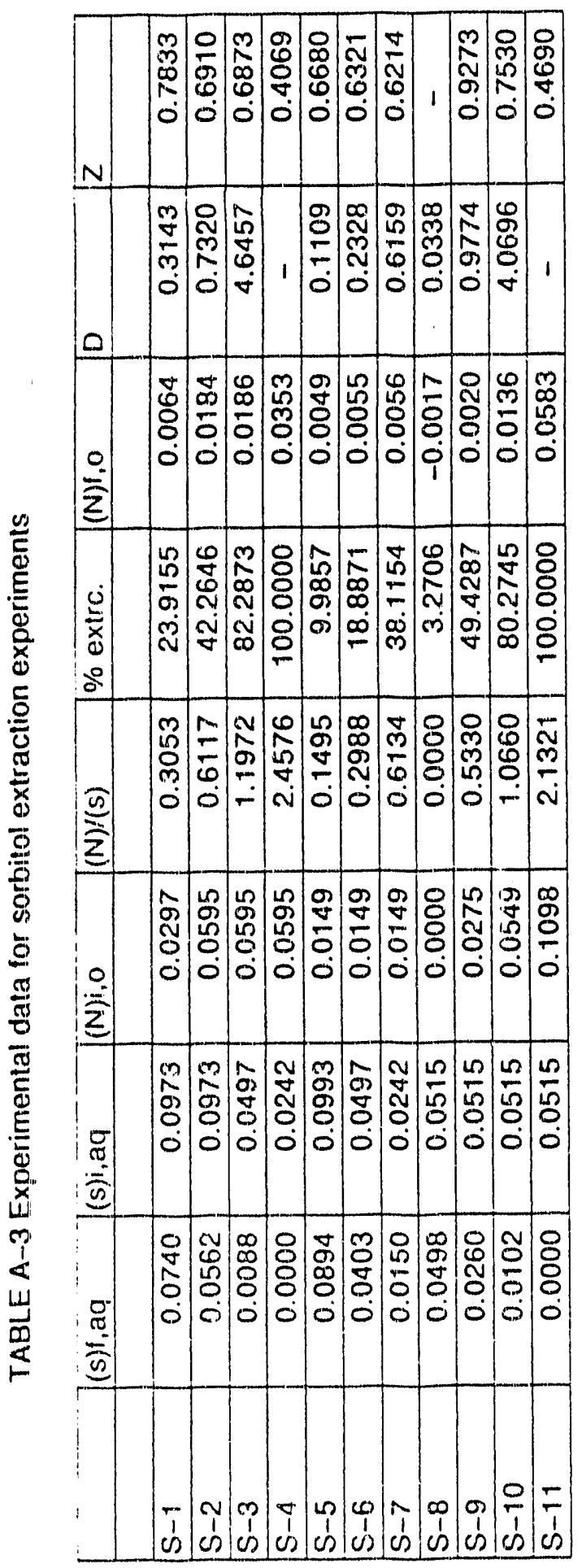



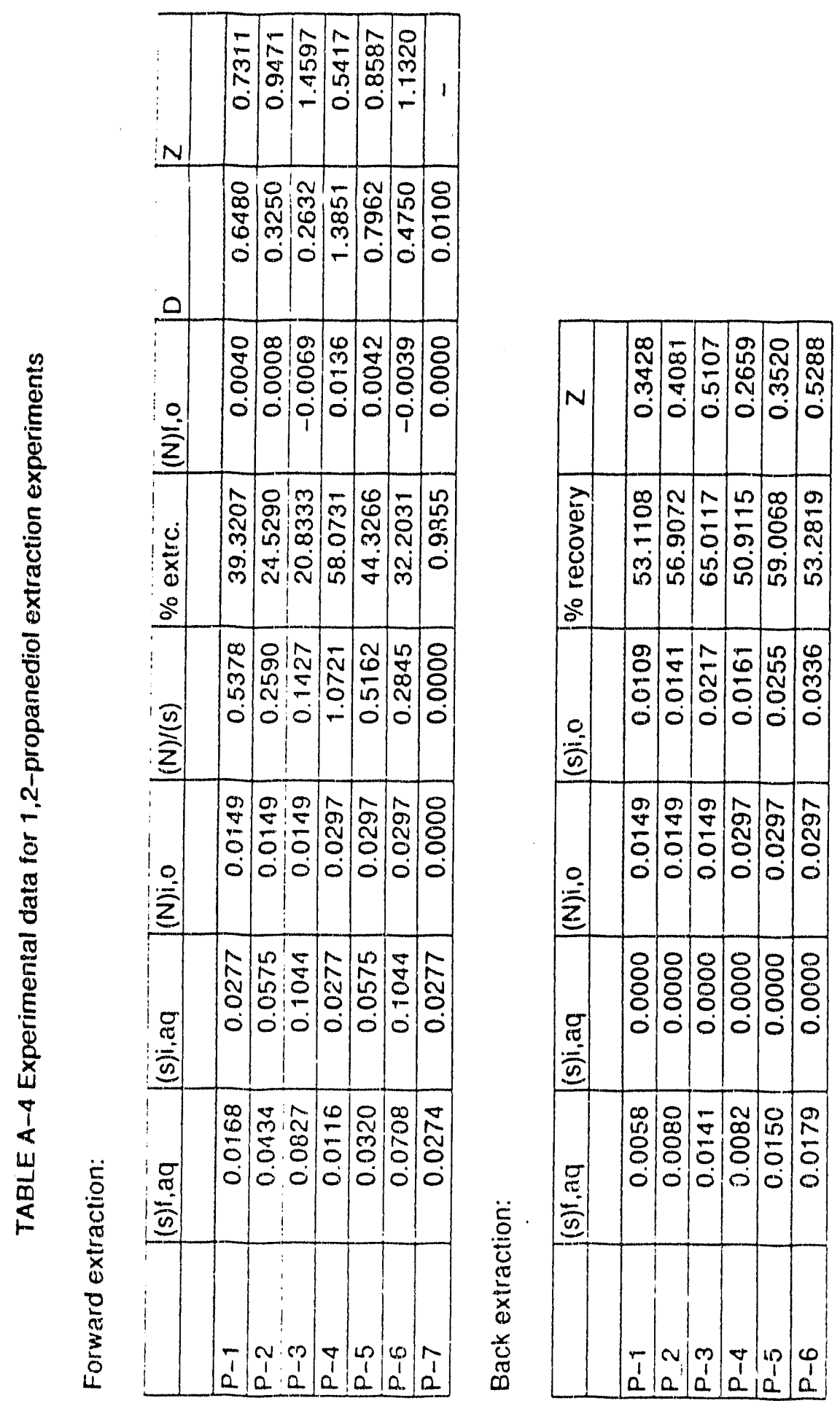


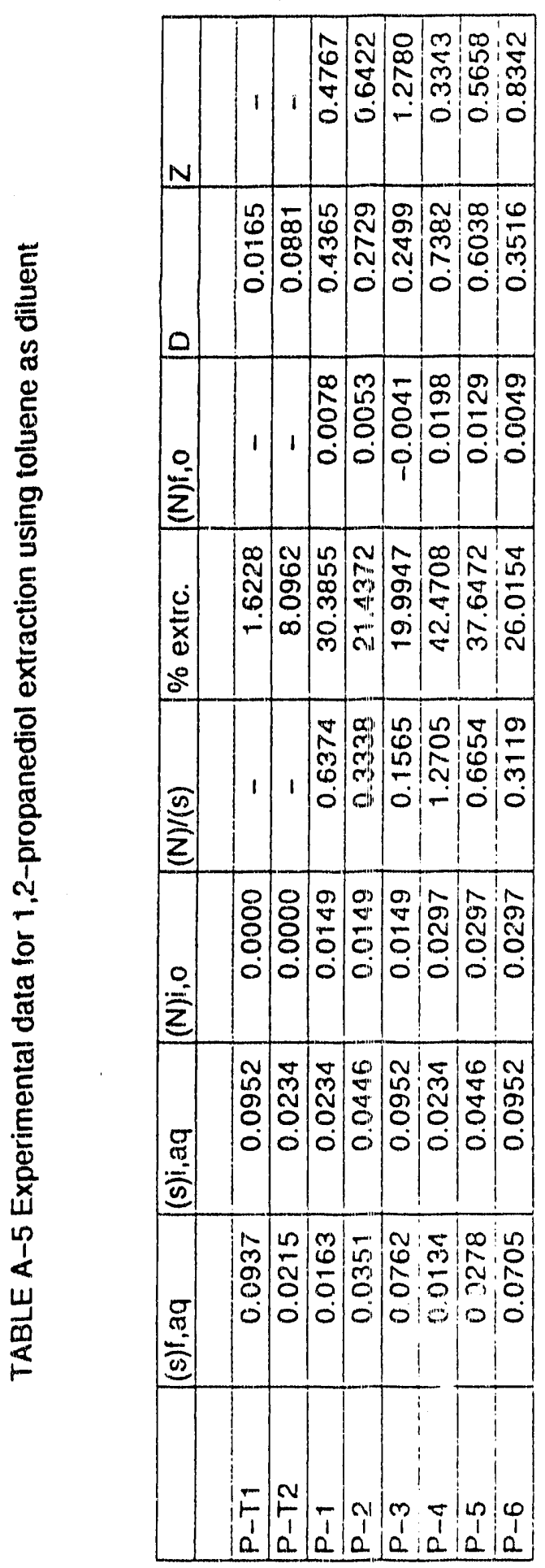




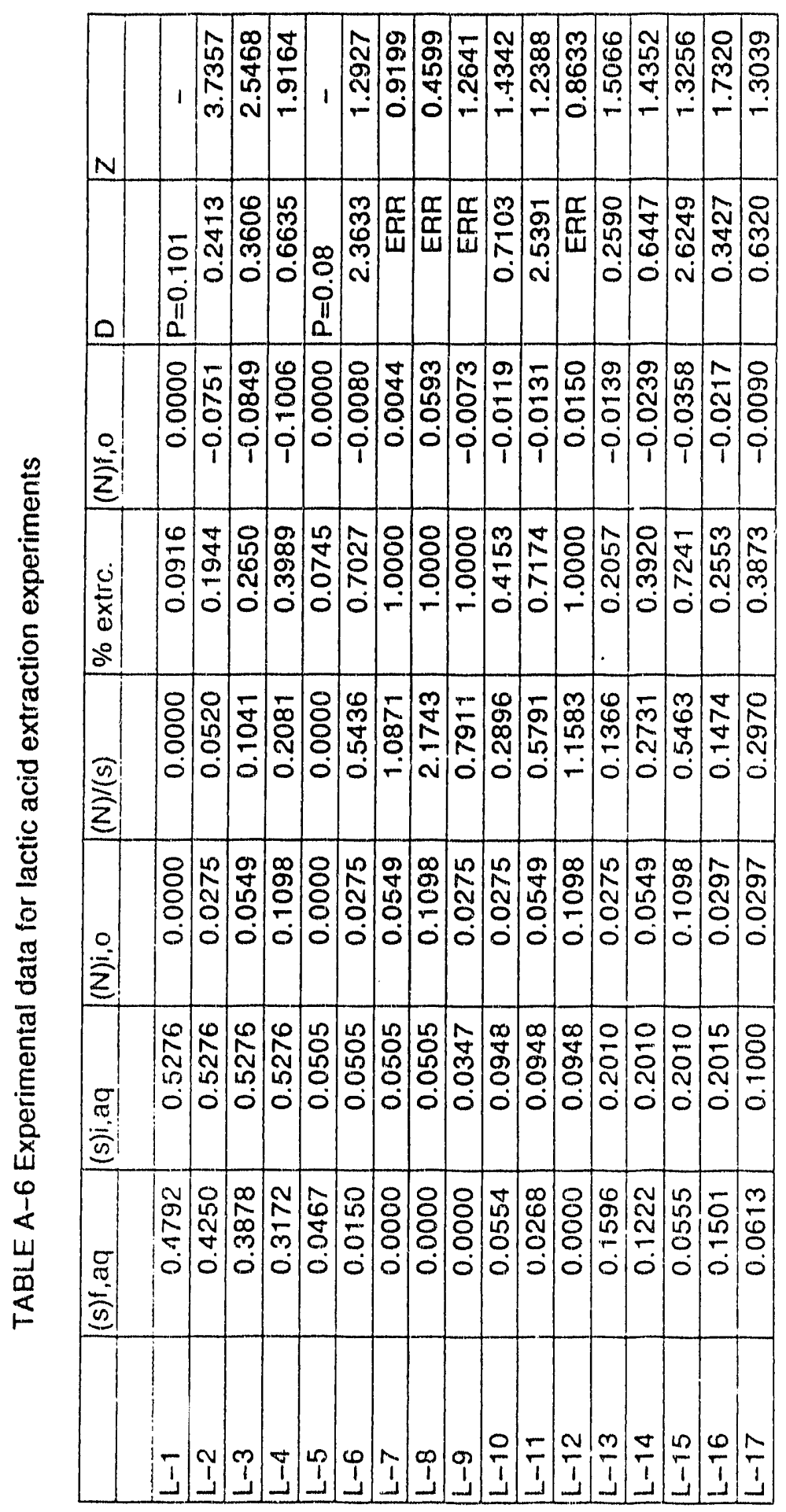



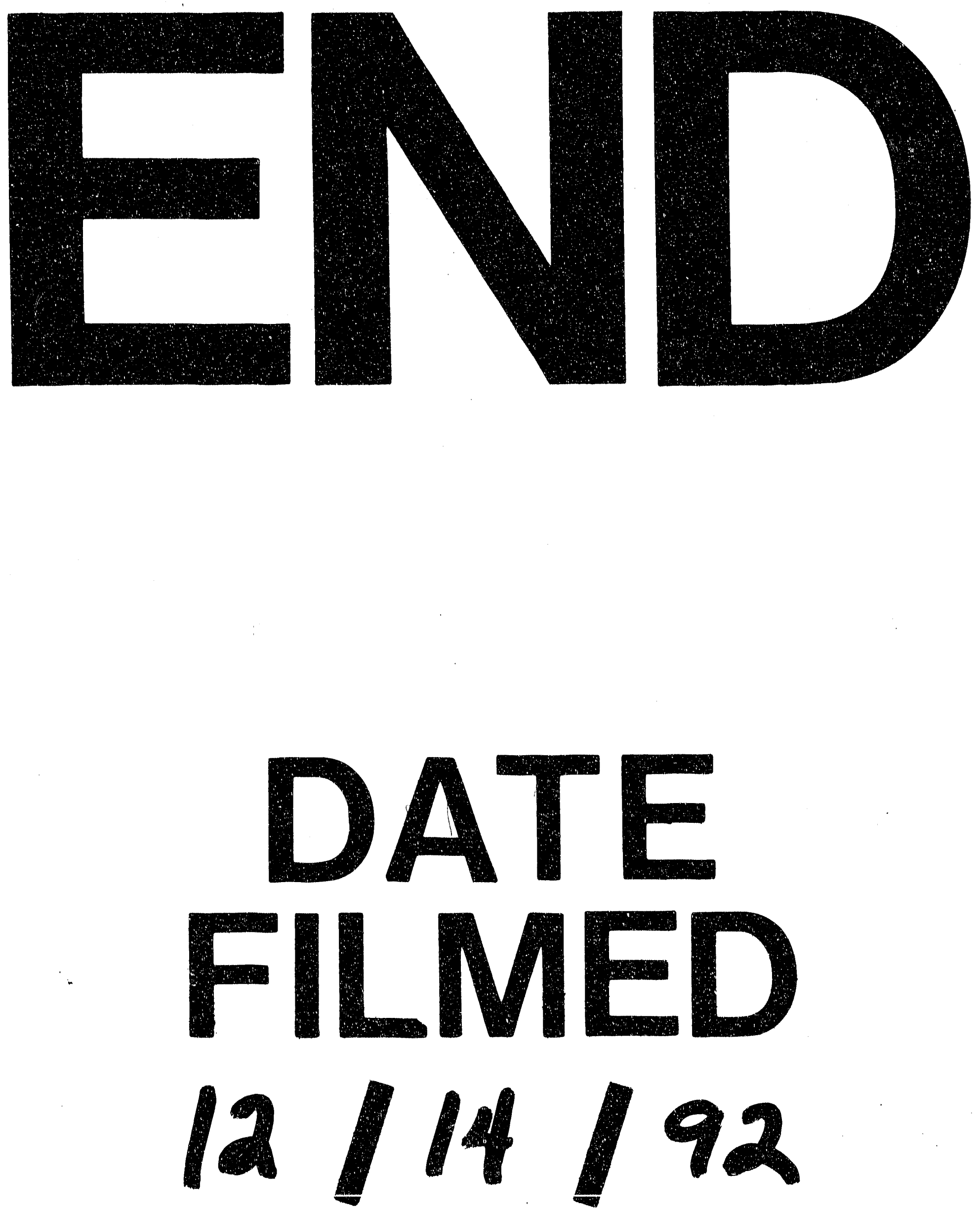


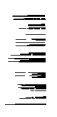

